\title{
Information Technology for Designing Rule bases of Fuzzy Systems using Ant Colony Optimization
}

\author{
OLEKSIY KOZLOV \\ Petro Mohyla Black Sea National University, 10 68th Desantnykiv st., Mykolaiv, 54003, Ukraine, (e-mail: kozlov_ov@ukr.net) \\ Corresponding author: Oleksiy Kozlov (e-mail: kozlov_ov@ukr.net).
}

\begin{abstract}
: ABSTRACT This paper proposes the universal information technology for designing the rule bases (RB) with the formation of optimal consequents for fuzzy systems (FS) of different types on the basis of ant colony optimization (ACO) techniques. The developed ACO-based information technology allows effectively synthesizing rule bases of various dimensions both for the MISO and MIMO fuzzy systems taking into account the particular features of the RB consequents formation in the conditions of insufficient initial information. In order to study and validate the efficiency of the presented information technology the design of the RB for the adaptive fuzzy control system of the ship steering device is carried out in this work. The computer simulations results show that adaptive control system with developed RB provides achievement of high enough quality indicators of rudder angle control. Thus, application of the proposed ACO-based information technology allows designing effective RB with optimal consequents by means of minor computational costs that, in turn, confirms its high efficiency.
\end{abstract}

: KEYWORDS fuzzy system; designing rule base; information technology; bioinspired multi-agent techniques; ant colony optimization; adaptive control system; ship steering device.

\section{INTRODUCTION}

$\mathrm{C}$ URRENTLY, fuzzy logic, fuzzy sets theory and soft computing techniques are widely being used when designing and researching computer systems of various classes [1-3]. After theoretical substantiation in a number of fundamental works by L. Zadeh, I. Mamdani, B. Kosko et al. [4-6] the mathematical apparatus of fuzzy logic is successfully used for solving different tasks in conditions of incomplete information and uncertainty. The most expedient is the use of fuzzy logic methods and means in the development of intelligent control and decision making systems for automation of complex nonstationary and nonlinear technical plants, in particular, such as ships and marine floating structures, unmanned underwater and aerial vehicles, mobile robots and drones, industrial robotic production lines, chemical reactors, power plants, etc. [7-9].

Initially fuzzy control systems were built mainly on the basis of expert knowledge and assessments that significantly limited their applications for automation of non-stationary plants or plants with uncertainties, for which there was no significant experience of manual control accumulated by their operators [5, 10, 11]. Also, fuzzy decision making systems used in various fields (medical and technical diagnostics, financial management, stock market forecasting, etc.) could not always operate enough accurately and efficiently, since the process of their design depended significantly on the qualifications and experience of developers, as well as on a number of other subjective factors [12-14]. However, in the past two decades numerous approaches, methods and information technologies have been propounded to develop highly efficient fuzzy systems (FSs) on the basis of certain structural-parametric optimization procedures that allow significantly reduce the negative influence of subjective factors on the process of fuzzy systems development, as well as significantly expand the scope of their applications [15-17]. Moreover, modern 
research in the field of creation and development of fuzzy decision making and control systems shows, that due to the intensive development and increase in the power of computer technology, bioinspired intelligent methods and information technologies of global search are quite promising for solving problems of synthesis and optimization of FSs of various configurations and purposes [18-20]. These bioinspired methods are stochastic methods of global optimization and have a number of advantages over classical search methods [21-23]. For the effective use of all the advantages of bioinspired approaches and methods of FS synthesis and optimization, it is necessary to develop and approbate appropriate advanced information technologies for their implementation, which is currently a rather complex and relevant issue.

This paper focuses on the development and research of an advanced information technology for solving such an urgent and complicated problem as the designing rule bases of fuzzy decision making and control systems based on the bioinspired intelligent methods, specifically ant colony optimization methods. The paper is organized in the following way. The statement of the research problem, brief literature survey in the studied area and the purpose of this work are presented in section 2. Section 3 describes in detail the proposed information technology of designing the rule bases. Section 4 , in turn, presents the results of studying the effectiveness of the developed information technology on a specific example of an adaptive fuzzy control system for ship steering device with a detailed analysis of the computer simulation results.

\section{PROBLEM STATEMENT AND RELATED WORKS}

Recently numerous papers have been published with examples of designing and successful application of fuzzy decision making and control systems of different types in various sectors of human activity [24-26]. For instance, in modern fuzzy control systems fuzzy inference devices can be used as controllers, identifiers, adaptive devices, observers, blocks for setting modes of the upper control level, etc. [27, 28]. The generalized MIMO (multiple inputs, multiple outputs) fuzzy system is shown in Fig. 1, where the following notations are accepted: $\mathrm{HE}$ is the human expert; FU is the fuzzification unit; DFU is the defuzzification unit; $x_{1}, x_{2}, \ldots, x_{i}, \ldots, x_{n}$ are the inputs of the fuzzy system; $y_{1}$, $y_{2}, \ldots, y_{j}, \ldots, y_{m}$ are the outputs of the fuzzy system;

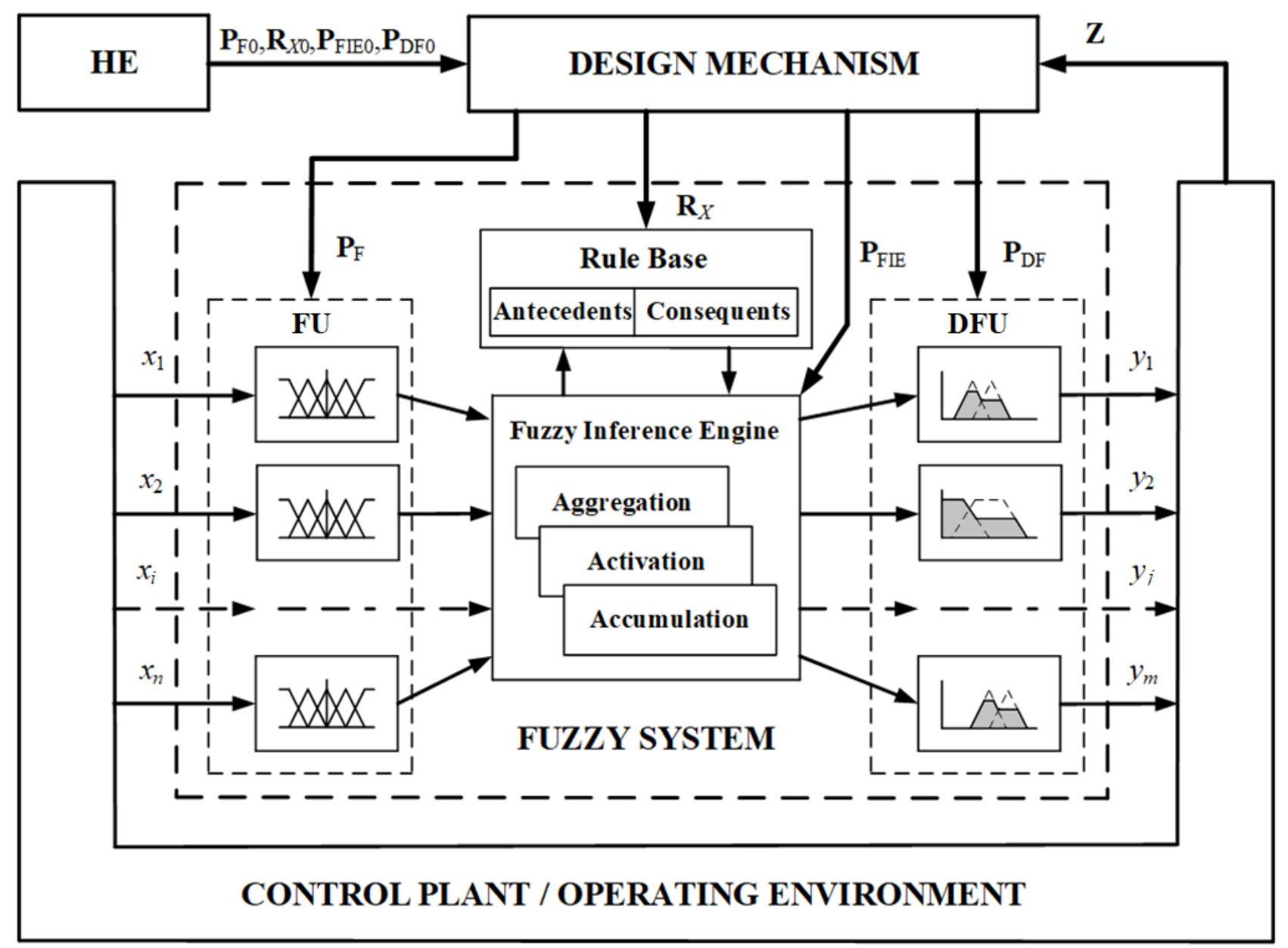

Figure 1. Structure of the generalized MIMO fuzzy system. 
$\mathbf{P}_{\mathrm{F}}$ is the vector that determines the number, types and parameters of linguistic terms (LT) of the system input variables; $\mathbf{R}_{X}$ is the rule base (RB) consequents vector; $\mathbf{P}_{\mathrm{FIE}}$ is the vector that defines the operations of aggregation, activation and accumulation of the fuzzy inference engine; $\mathbf{P}_{\mathrm{DF}}$ is the vector that determines the number, types and parameters of linguistic terms of the FS output variables, as well as the defuzzification method; $\mathbf{Z}$ is the vector of output variables of the control plant / operating environment used in designing process of the given fuzzy system.

The generalized fuzzy MIMO system shown in Fig. 1, implements the nonlinear dependence $f_{\mathrm{FS}}$ [29]

$$
\begin{gathered}
\mathbf{Y}=f_{\mathrm{FS}}(\mathbf{X}), \mathbf{Y}=\left(y_{1}, y_{2}, \ldots, y_{j}, \ldots, y_{m}\right), \\
\mathbf{X}=\left(x_{1}, x_{2}, \ldots, x_{i}, \ldots, x_{n}\right),
\end{gathered}
$$

where $\mathbf{X}$ is the vector of input variables $x_{1}, x_{2}, \ldots, x_{i}, \ldots, x_{n}$; $\mathbf{Y}$ is the vector of output variables $y_{1}, y_{2}, \ldots, y_{j}, \ldots, y_{m}$ of the presented fuzzy system.

The fuzzification unit determines the membership degree for the numerical values of all $n$ variables of the vector $\mathbf{X}$ to the corresponding input LTs of the considered fuzzy system [30]. The fuzzy inference engine, in turn, based on fuzzified signals and data received from the rule base, sequentially performs aggregation, activation and accumulation operations [31]. The rule base consists of a set of rules composed of defined antecedents and consequents. So, for example, to implement a functional dependence (1) by the presented MIMO fuzzy system, one of its rules built on the basis of Mamdani type fuzzy inference using the corresponding 4 linguistic terms for any variable $x_{i}(i=1 \ldots n)$ or $y_{j}(j=1 \ldots m)$ can be represented by the expression (2) [32]

$$
\begin{aligned}
& \text { IF " } x_{1}=A_{1} \text { ” AND " } x_{2}=A_{2} \text { " AND ... } \\
& \text { AND " } x_{i}=A_{3} \text { " ... AND } \ldots \text { AND " } x_{n}=A_{4} \text { " } \\
& \text { THEN " } y_{1}=B_{1} \text { ” AND " } y_{2}=B_{2} \text { "AND... } \\
& \text { AND " } y_{j}=B_{3} \text { ”... AND... AND" } y_{m}=B_{4} \text { ", }
\end{aligned}
$$

where $A_{1}, A_{2}, A_{3}, A_{4}, B_{1}, B_{2}, B_{3}, B_{4}$ are certain linguistic terms of FS inputs and outputs.

The defuzzification unit transforms the consolidated fuzzy inference into a crisp numerical signal for each FS output variable [31].

It is advisable to implement the design of the presented fuzzy system (Fig. 1) in an automated mode using the builtin designing mechanism. Wherein, the given designing mechanism should determine such vectors $\mathbf{P}_{\mathrm{F}}, \mathbf{R}_{X}, \mathbf{P}_{\mathrm{FIE}}$ and $\mathbf{P}_{\mathrm{DF}}$, at which a sufficiently effective use of the FS will be provided for solving a particular problem (for example, the problem of decision-making, automatic control, etc.). In this case, the efficiency of the fuzzy system can be estimated using a certain objective function $J$, which is calculated on the basis of the obtained values of the vector of measured output variables of the control plant or operating environment $\mathbf{Z}$ [29, 33]. Also, FS design and performance evaluation can be carried out on the basis of training samples and other experimental data. Herewith, the human expertoperator sets only the initial values (initial hypotheses) of the FS vectors $\mathbf{P}_{\mathrm{F} 0}, \mathbf{R}_{X 0}, \mathbf{P}_{\mathrm{FIEO}}$ and $\mathbf{P}_{\mathrm{DF} 0}$, that determine the initial structure, parameters and rule base.

The quality and productivity of the fuzzy system designing process directly depend on the methods and information technologies used to optimize its structure and parameters, implemented in the design mechanism [33, 34]. In turn, a number of studies at the moment are underway towards developing of different approaches, methods and information technologies for synthesis, parametric and structural optimization of fuzzy systems, the results of which are presented in publications [35-37]. In particular, information technologies and methods of FSs structural optimization on the basis of the optimal selection of the linguistic terms membership functions, reduction and interpolation of rule bases, procedures of defuzzification, etc. are presented in [38-40]. Also, quite many published papers are dedicated to the designing methods that include optimization of the LT parameters of the Mamdani FSs, and finding optimal weighting factors for the rule consequents of systems of Takagi-Sugeno type [41-43].

The results obtained in recent studies indicate that progressive bioinspired intelligent methods and information technologies are quite effective and promising for conducting design and training of different types fuzzy decision making and control systems [44-46]. The given bioinspired methods are usually divided into two main groups: (a) multi-agent methods, which mimic the social behavior of collective animals, insects and microorganisms and include methods of ant colony optimization [47], bee colony optimization [48], particle swarm optimization [49], bacterial foraging optimization [50], etc.; (b) evolutionary methods, that model processes of natural selection and evolution in nature and to which relate genetic methods [51], evolutionary strategies [52], methods of differential evolution [53], biogeography based optimization [54], artificial immune systems [55] and others. All these presented above methods and techniques relate to the methods of stochastic global optimization, that have a number of advantages compared to the conventional optimization approaches: (a) allow effectively optimizing large dimension FSs; (b) provide the opportunity for detailed search of large, nonsmooth and multimodal search space, excluding local optima entrapment; (c) do have any restrictions for the training data and FS objective functions $[18,21,22]$.

When applying the given bioinspired methods for solving the problem of structural-parametric design of a Mamdanitype fuzzy decision making and control systems, the task of automatic rule base development with the determination of optimal consequents at insufficient initial information deserves a special attention [29]. 
The rules antecedents of the RB are various combinations of linguistic terms of FS input variables $[44,56]$. In turn, the rules consequents are the certain sets of LTs of the system output variables. Wherein, in each $r$-th rule of $\mathrm{RB}(r=1,2$, $\ldots, s$, where $s$ is the total amount of rules in RB) the consequent's part $L T_{r j}$ that corresponds to each $j$-th output variable $(j=1,2, \ldots, m)$ is selected from the corresponding $j$-th set of terms $\left\{L T_{1 j}, L T_{2 j}, \ldots, L T_{v j}\right\}$, which includes all $v_{j}$ linguistic terms of the given FS output variable $y_{\mathrm{j}}$

$$
L T_{r j} \in\left\{L T_{1 j}, L T_{2 j}, \ldots, L T_{v j}\right\}
$$

For example, for the fuzzy system with three output variables $(m=3)$ the following numbers of linguistic terms are chosen for the given output variables: $v_{1}=3, v_{2}=7$ and $v_{3}=5$. In this case, the following sets of the output variables LTs can be formed:

$$
\begin{gathered}
L T_{r 1} \in\left\{L T_{11}, L T_{21}, L T_{31}\right\}=\{\mathrm{N}, \mathrm{Z}, \mathrm{P}\} ; \\
L T_{r 2} \in\left\{L T_{12}, L T_{22}, L T_{32}, L T_{42}, L T_{52}, L T_{62}, L T_{72}\right\}= \\
\{\mathrm{BN}, \mathrm{N}, \mathrm{SN}, \mathrm{Z}, \mathrm{SP}, \mathrm{P}, \mathrm{BP}\} ; \\
L T_{r 3} \in\left\{L T_{13}, L T_{23}, L T_{33}, L T_{43}, L T_{53}\right\}= \\
\{\mathrm{BN}, \mathrm{SN}, \mathrm{Z}, \mathrm{SP}, \mathrm{BP}\},
\end{gathered}
$$

where $\mathrm{BN}$ is big negative; $\mathrm{N}$ is negative; $\mathrm{SN}$ is small negative; $\mathrm{Z}$ is zero; $\mathrm{SP}$ is small positive; $\mathrm{P}$ is positive; $\mathrm{BP}$ is big positive.

The consequents vector $\mathbf{R}_{X}$ may be synthesized in various ways, thus the task of the consequents optimization is the task of selecting the optimal consequents vector $\mathbf{R}_{\text {opt }}$ from the set of all existing alternative variants that provides high (optimal) performance of a fuzzy control or decision making system [57]. In general terms the rule base consequents vector $\mathbf{R}_{X}$ for the $x$-th alternative variant is represented as follows [29]:

$$
\begin{gathered}
\mathbf{R}_{X}=\left\{L T_{X 11}, L T_{X 12}, \ldots, L T_{X 1 j}, \ldots, L T_{X 1 m} ;\right. \\
L T_{X 21}, L T_{X 22}, \ldots, L T_{X 2 j}, \ldots, L T_{X 2 m} ; \ldots ; \\
L T_{X r 1}, L T_{X r 2}, \ldots, L T_{X r j}, \ldots, L T_{X r m} ; \ldots ; \\
\left.L T_{X s 1}, L T_{X s 2}, \ldots, L T_{X s j}, \ldots, L T_{X s m}\right\}, \\
L T_{X r j} \in\left\{L T_{1 j}, L T_{2 j}, \ldots, L T_{v j}\right\}, \\
j=\{1,2, \ldots, m\}, r=\{1,2, \ldots, s\} .
\end{gathered}
$$

The task of finding the optimal vector of consequents $\mathbf{R}_{\mathrm{opt}}$ is a complicated discrete optimization task of large dimension, that may be successfully solved by means of an effective information technology developed on the basis of bioinspired global search algorithm and that takes into account all features of the consequents synthesis of the rule base at the information uncertainty or/and insufficient initial information [29, 57]. Contemporary studies show that ant colony optimization methods and algorithms have proven themselves to be quite promising and more effective in comparison with other bioinspired optimization approaches at solving tasks of discrete optimization of large dimension [29, 47, 58]. So, a number of published papers considered examples of the efficient application of different ant colony optimization (ACO) techniques to solve various problems of discrete optimization: the traveling salesman challenge [59], the problem of graph coloring [47], the scheduling problem [60], the problem of optimizing the vehicles routes [61], etc. [62, 63]. Also, results of successful implementation of ACO techniques for optimizing different types of fuzzy systems were given in $[64,65]$. In particular, in papers [29, 64] ACO-based information technologies were developed and presented for synthesis and optimization of rule bases for MISO (multiple inputs, single output) fuzzy systems. The main disadvantage of these information technologies is their limited use only for MISO fuzzy systems. Also, with an increase in the dimension of the optimized rule bases, the computational costs of these technologies increase significantly and their applications become impractical. Thus, development of a universal information technology based on the ACO methods that will make it possible to effectively synthesize rule bases of various dimensions both for the MISO and MIMO fuzzy systems considering the main features of the $\mathrm{RB}$ consequents formation in the conditions of insufficient initial information is a rather urgent task.

The purpose of this work is development and research of the universal information technology for designing rule bases with optimal consequents forming for different types fuzzy systems based on ant colony optimization techniques.

\section{ACO-BASED INFORMATION TECHNOLOGY FOR} DESIGNING RULE BASES

The methods of ant colony optimization are based on modeling the social behavior of ants, that belong to one large colony, in the process of the collective solving the problem of finding the shortest path to the foraging source [29, 47, 65]. The real ants in the colony are simulated by interacting agents of the joint self-organizing system that travel along the graph of the problem solutions with the aim of finding the best (optimal) solution [58, 64]. The length of the traversed path of each artificial ant is expressed by a certain value of the objective (cost) function $J$ that measures the performance of the developed solution to the problem. Thus, the ACO methods can be effectively applied to solve the problems described by a graph consisting of nodes and edges connecting the nodes [47]. The idea of applying the ACO principles in the information technology for designing rule base is to represent the structure of the fuzzy system RB as a specific graph, on the edges and nodes of which the agentsants will move [29]. The route of every $z$-th agent of the colony runs through certain edges and nodes of the given graph at every separate iteration with number $N$. After each $z$-th agent passes through the whole graph at every $N$-th 
iteration the corresponding RB of fuzzy system with a certain consequents vector $\mathbf{R}^{z}(N)\left(z=1, \ldots, z_{\max } ; N=1, \ldots, N_{\max }\right)$ is formed. The route length that a certain ant has travelled in the current iteration depends on the value of the FS objective function $J^{z}(N)$.

In previous studies [29, 64], the following concept of graph construction for RB development with optimal consequents for MISO fuzzy systems was used. The RB graph (Fig. 2) include one zero layer, which has only one node - the point of the route starting for all $z_{\max }$ colony ants, and $s$ main layers. In turn, every main layer has a serial number $r$ and determines the corresponding $r$-th rule $(r=1$,
$2, \ldots, s)$ of the rule base. Moreover, every $r$-th layer of the graph has $v$ nodes, that correspond to all rules consequents and, respectively, to all linguistic terms of the fuzzy system single output. Every $r \gamma$-th node of the $r$-th layer determines $\gamma$-th consequent $(r=1,2, \ldots, s ; \gamma=1,2, \ldots, v)$ that can be selected for the $r$-th rule of the developed fuzzy rule base [29]. In addition, every node in each layer (except zero layer) of the given RB graph is connected by edges with every node of the previous and subsequent layers. As for the only node of the zero layer, it is connected only with each node of the first layer.

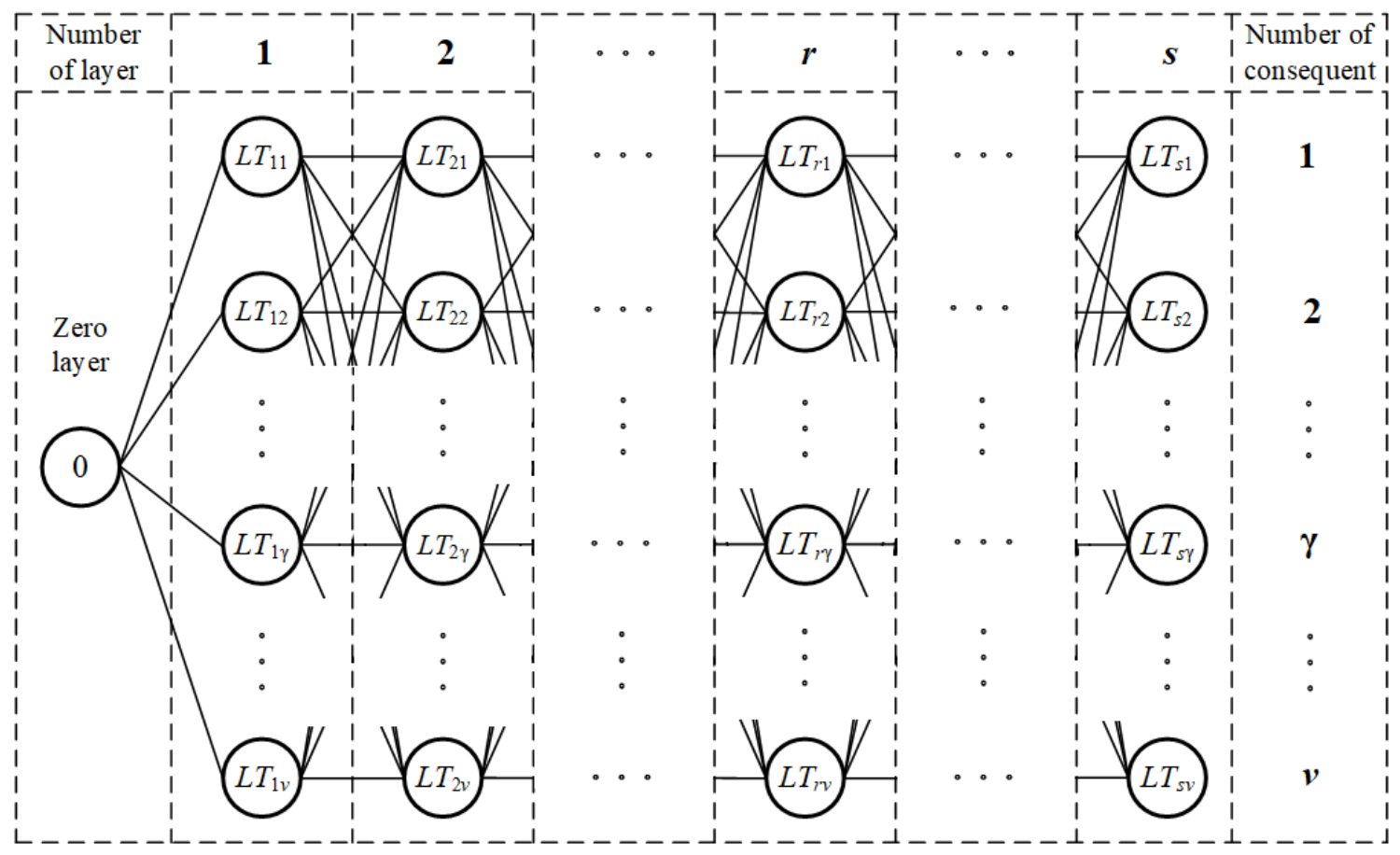

Figure 2. Rule Base Graph of the RL-type.

Since, in this graph each $r$-th rule of the rule base corresponds to a specific $r$-th layer, the concept of constructing a graph of such type can be called "Rule-Layer", and the graph itself - a RL-type graph, in turn.

Being that, in ACO algorithms most of the operations (movement of ants, pheromone increasing, evaporation and updating) at each iteration are performed with the edges of the graph, the number of edges in the graph significantly affects the computational complexity and speed of these algorithms. Therefore, the number of edges $q$ for the graph of the RL-type can be calculated by the following equation

$$
q=v^{2}(s-1)+v
$$

So, for instance, for a certain FS with a rule base of small dimension with 25 rules $(s=25)$ and with 7 LTs $(v=7)$ that its output variable has, the edges number of the graph according to equation (6) is equal to 1183 . In this case, for such a number of graph edges $(q=1183)$, the implementation of information technology for designing RB based on such a concept will already require significant computational and time costs.

When using this concept of graph constructing for designing RB of the MIMO fuzzy systems with $m$ output variables the number of edges $q$ will be calculated by the equation (7)

$$
q=\sum_{j=1}^{m} v_{j}^{2}(s-1)+v_{1} .
$$

In this case, for a similar fuzzy system with 25 rules $(s=$ $25)$ in the rule base that has 3 outputs $(m=3)$ with 7 linguistic terms for each $\left(v_{1}=7, v_{2}=7, v_{3}=7\right)$, the number of graph edges according to equation (7) is equal to 3535. With such number of graph edges $q$ and grater, the usage of the given ACO-based information technology will require excessive computational and time costs, which in some cases will be inappropriate. 
Thus, the application of the developed in previous works $[29,64]$ "Rule-Layer" concept of constructing the RB graph does not allow effectively using the ACO-based approach when developing MIMO fuzzy systems. Moreover, when developing rule bases of a sufficiently large dimension, the computational costs can be so significant that the application of this concept even for MISO FSs will no longer be advisable.

To significantly reduce the number of edges $q$ in the graph, which will substantially decrease the computational costs of information technology and, accordingly, increase its performance, this paper proposes the new concept for constructing a rule base graph, which can be called "RuleNode". Application of this concept in conjunction with the principles of ACO-based search gives the opportunity to create the universal information technology that allows synthesizing rather complex rule bases for both MISO and
MIMO fuzzy systems with rather high efficiency.

The generalized RB graph built on the basis of the concept "Rule-Node" is presented in Fig. 3. In a graph of the $\mathrm{RN}$-type built according to this concept, each $r$-th rule will correspond to $m$ sequentially connected nodes with the numbers: $r 1, r 2, \ldots, r j, \ldots, r m$. In general, for the MIMO fuzzy system with $m$ outputs, each of which has $v_{j}(j=1,2$, $\ldots, m)$ linguistic terms, the given rule base graph will have one zero node, that is a point for route starting of all ants, and $s m$ main nodes, sequentially connected by means of edges (arcs). In turn, each $r j$-th node $(r=1,2, \ldots, s ; j=1,2, \ldots, m)$ is connected to the next node on the right $r(j+1)$ by means of $v_{j+1}$ arcs, that correspond to all $v_{j+1}$ LTs of the fuzzy system output $y_{j+1}$ with the number $j+1$. Thus, each $\gamma$-th linguistic term $\left(\gamma=1,2, \ldots, v_{j}\right)$ of the $j$-th FS output variable $(j=1,2$, $\ldots, m)$ that can be chosen as a consequent's part for the $r$-th $\mathrm{RB}$ rule $(r=1,2, \ldots, s)$ corresponds to $r \gamma j$-th arc of the graph.

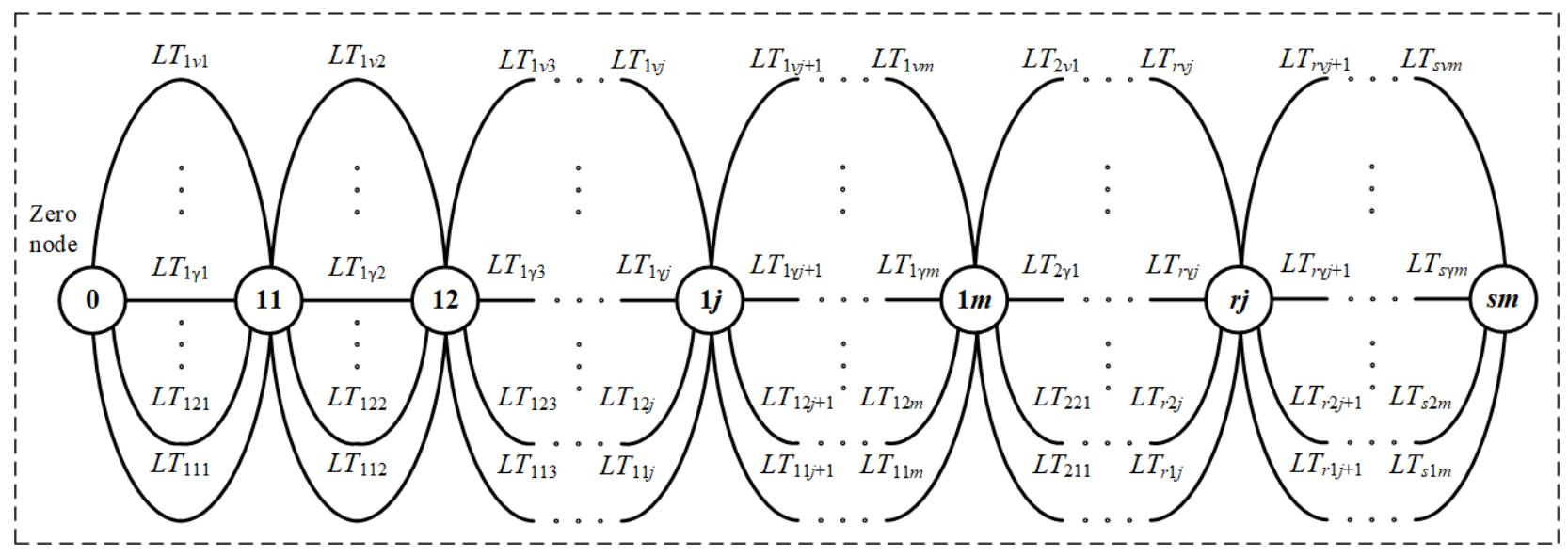

Figure 3. Rule Base Graph of the RN-type.

In this case, the total number of edges $q$ in the RN-type graph is determined by the sum of linguistic terms of all $m$ output variables of the system multiplied by the total number of rules $s$

$$
q=\sum_{j=1}^{m} v_{j} s
$$

For the given above example of the MIMO fuzzy system with 25 rules and 3 outputs with 7 linguistic terms for each, the number of RN-type graph arcs will be equal to 525, which is more than 6 times less than for RL-type graph built for this system. Also, using the "Rule-Node" concept at the graph building for the RB of MISO fuzzy system, each rule corresponds to only one node and not to a layer of nodes as in the RL-type graph.

Thus, in this work, at developing the universal information technology for designing RB of both MISO and MIMO fuzzy systems with enough high efficiency, the proposed "Rule-Node" concept of constructing the rule base graph is used. Next, let us consider the main operations of the ACO method used in this information technology for RB development.

During the movement of all the ants along the graph, the probability $P_{r \gamma j}^{z}(N)$ of the $z$-th agent passing along the $r \gamma j$ th arc from the node with number $r(j-1)$ to the $r j$-th node ( $\left.r \in\{1, \ldots, s\} ; \quad \gamma \in\left\{1, \ldots, v_{j}\right\} ; \quad j \in\{1, \ldots, m\}\right)$ in the $N$-th iteration $\left(N=1, \ldots, N_{\max }\right)$ is calculated based on the equation [29]:

$$
P_{r \gamma j}^{z}(N)=\frac{\left[\tau_{r \gamma j}(N)\right]^{\alpha} \cdot\left[\eta_{r \gamma j}\right]^{\beta}}{\sum_{r \gamma j j=r l j}^{r v j}\left[\tau_{r \gamma j}(N)\right]^{\alpha} \cdot\left[\eta_{r \gamma j}\right]^{\beta}},
$$

where $\tau_{r \gamma j}(N)$ is the pheromone intensity on the $r \gamma j$-th arc at the iteration $N ; \eta_{r \gamma j}$ is an inverse of the relative length $D_{r \gamma j}$ of the $r \gamma j$-th arc; $\alpha$ is an adjustable parameter which determines the importance of the pheromone trace considering on the arc; $\beta$ is the parameter that regulate importance of the relative length $D_{r \gamma j}$ of the $r \gamma j$-th arc.

The parameter $\eta_{r \gamma j}$ is calculated as follows 


$$
\eta_{r \gamma j}=\frac{1}{D_{r \gamma j}}
$$

In turn, the value of the relative length $D_{r \gamma j}$ for each $r \gamma j$-th arc can be set based on the knowledge of experts or any preliminary known information (if available) about the system or the problem being solved. In this case, the more appropriate the given $r \gamma j$-th linguistic term is considered for the $j$-th variable in the $r$-th rule, the smaller the value of the arc relative length $D_{r \gamma j}$ is set. In the absence of any preliminary information the values of the relative length for all arcs can be set randomly or in some other way.

After each $z$-th agent travels through certain graph arcs of all $s m$ nodes on every $N$-th iteration, the path length of the given agent for synthesized consequents vector $\mathbf{R}^{z}(N)$ is then calculated that corresponds to the certain value of the objective function $J^{z}(N)$ for the fuzzy system. For example, if the rule base of a given system is designed on the basis of a training sample, consisting of certain sets of values of input and output variables the mean squared error (MSE) may be used as an objective function that measures the average of the squares of the errors between the estimated and actual output values of the FS. In the case of the designing RB for the fuzzy control system the objective function may be presented as an integral quadratic error of control or the integral quadratic deviation between the outputs of the reference model and the real control plant [29]. In turn, for the fuzzy decision making system the objective function may be calculated as the percentage of wrong decisions obtained for the test dataset.

The amount of pheromone left on each $r \gamma j$-th arc by the $z$-th agent at the $N$-th iteration, is calculated using the current value of the objective function $J^{z}(N)$ [64] according to the following expression:

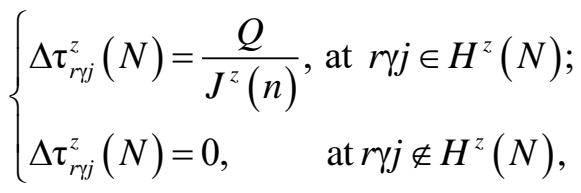

where $Q$ is the constant that impact on the amount of pheromone that will be left by an ant on its route; $H^{z}(N)$ is a set of arcs that $z$-th ant has already passed at the $N$-th iteration. In turn, the value of the parameter $Q$ is usually set to be of one order with the optimal value of the objective function $J_{\text {opt }}$ The pheromone amount $\Delta \tau_{r \gamma j}^{z}(N)$ left by $z$-th agent on the arc $r \gamma j$ at the $N$-th iteration inversely proportional depends on the objective function value $J^{z}(N)$. For instance, if the objective function is small, then the pheromone has a high concentration, and vice versa.

When the pheromone values $\Delta \tau_{r \gamma j}^{z}(N)$, left by each $z$-th ant on each $r \gamma j$-th arc of the graph, are calculated based on the equation (11), they are used for increasing the pheromone total amount on the graph's arcs [29] based on the equation (12)

$$
\tau_{r \gamma j}(N)=\tau_{r \gamma j}(N-1)+\rho \cdot \sum_{z=1}^{z_{\max }} \Delta \tau_{r \gamma j}^{z}(N)
$$

where $\rho$ is the coefficient that enhances the pheromone amount left by ants on their routes (in most cases it is set in the range $0 \ldots 1$ ).

In order to increase the convergence speed of the search process in the proposed information technology it is advisable to implement the elite strategy, due to to which elite ants are used in the search process [29, 47]. According to this strategy an additional pheromone increasing is implemented at each iteration to these arcs, which are parts of the best route at the current iteration, that provide the least objective function $J_{\min }(N)$. The given pheromone amount that is left on each arc of the best route at $N$-th iteration, is calculated on the basis of equation (13) using the number of elite ants $e$ and the objective function value $J_{\min }(N)$

$$
\Delta \tau_{e}(N)=\frac{e \cdot Q}{J_{\min }(N)}
$$

After pheromone increasing using equations (12) and (13) at each $N$-th iteration for each $r \gamma j$-th arc of the graph the operation of pheromone evaporation [29] is applied in accordance with expression

$$
\tau_{r \gamma j F}(N)=\tau_{r \gamma j}(N) \cdot(1-\rho)
$$

where $\tau_{r \gamma j F}(N)$ is a final amount of the pheromone on the $r \gamma j$ th arc in the end of $N$-th iteration after implementation of the procedure of evaporation.

At transition to the iteration $N+1$ from the $N$-th iteration $\left(N=1, \ldots, N_{\max }\right)$ on each $r \gamma j$-th graph's arc $(r \in\{1, \ldots, s\}$; $\left.\gamma \in\left\{1, \ldots, v_{j}\right\} ; j \in\{1, \ldots, m\}\right)$, the amount of pheromone is updated using equation (15)

$$
\tau_{r \gamma j}(N+1)=\tau_{r \gamma j F}(N) .
$$

Thus, the proposed ACO based information technology for the designing rule bases with formation of optimal consequents for FSs of different types consists of the next successive stages.

Stage 1. The ACO based information technology initialization. At this stage, the RN-type graph is formed, structure of which corresponds to the RB of the designed fuzzy system. In turn, the graph consists of one zero node and $s m$ main nodes, sequentially connected by arcs, the total number of which $q$ is determined by the sum of linguistic terms of all $m$ output variables multiplied by the total number of rules $s$. In this case, rule base can be full with the number of rules $s$ corresponding to all possible combinations of linguistic terms of the $n$ input variables, or reduced. At developing the RB antecedents automatically, the number of 
rules $s$ will be full. In turn, at composing the rule base antecedents on the basis of the experts' knowledge some antecedents can be previously excluded if there is preliminary information about their insignificant effect on the system operation, and, in this case, the RB will be reduced. Also at this stage, the objective function $J$ and its optimal value $J_{\text {opt }}$ are set. Moreover, an ant colony is created and the main adjustable parameters of the ACO algorithm are set: the number of ants in the colony $z_{\max }$, coefficients $\alpha$, $\beta, Q, \rho$, maximum iterations number $N_{\max }$, as well as the number of elite ants $e$. Moreover, the values of the relative lengths $D$ and initial small amount of the pheromone $\tau$ are set for all arcs before the ants begin to move at this stage.

Stage 2. Ants movement along the graph nodes of the fuzzy system RB. Moving of every $z$-th ant of the colony, which is created at Stage 1, starts from the zero node and is sequentially performed through all $\mathrm{sm}$ main graph's nodes from left to right. The transitions number for every $z$-th ant is the same as total number of nodes $s m$ of the graph. If $z$-th ant is placed at the node $r(j-1)$, then the probability $P_{r \gamma j}^{z}(N)$ of its transition to the node $r j$ through the $r \gamma j$-th arc is calculated by means of the expression (9). In turn, the inverse $\eta_{r \gamma j}$ of the relative length $D_{r \gamma j}$ of the $r \gamma j$-th arc is calculated based on equation (10). Stage 2 continues until every $z$-th ant of the colony passes all $\mathrm{sm}$ main nodes of the graph.

Stage 3. Estimation of the generated vectors of the RB consequents. At this stage the fuzzy system objective function $J^{z}(N)$ is calculated for each consequents vector of the RB rules $\mathbf{R}^{z}(N)$, generated by every $z$-th ant in the colony $\left(z=1, \ldots, z_{\max }\right)$ during its moving at Stage 2.

Stage 4. Checking for completion of the design process. At this stage, the best vector of RB consequents $\mathbf{R}_{\text {best }}(N)$, generated by the ants routes at Stage 2, is selected, that has the least objective function $J_{\min }(N)$ of fuzzy system. For this best vector of RB consequents $\mathbf{R}_{\text {best }}(N)$ the design process completion is checked, that is determined by achieving the objective function optimal value $\left(J_{\min }(N) \leq J_{\mathrm{opt}}\right)$ or by implementation of the maximum possible number of iterations $N_{\max }$. The optimization process can be also considered to be complete if the fuzzy system objective function $J$ does not decrease during a certain (previously set) number of iterations. In case of the positive check, the transition to Stage 8 is carried out. Otherwise, transition is done to Stage 5.

Stage 5. Pheromone increasing on the graph arcs of the fuzzy system RB. At this stage, the pheromone amount, left by every $z$-th ant $\left(z=1, \ldots, z_{\max }\right)$ during moving at Stage 2 on every $r \gamma j$-th arc, is calculated based on (11) using objective function values $J^{z}(N)$, calculated at Stage 3. The calculated values of the pheromone amount $\Delta \tau_{r \gamma j}^{z}(N)$, left by each $z$-th ant on each $r \gamma j$-th arc when moving, are used for pheromone increasing on the graph arcs on the basis of equation (12). Also, the elite strategy is used for the additional pheromone increasing at this stage for the arcs, that are parts of the best route at the current iteration with the least objective function value $J_{\min }(N)$. The given additional amount of pheromone value is determined by the expression (13).

Stage 6. Pheromone evaporation on the RB graph arcs. For every $r \gamma j$-th arc of the RB graph the evaporation procedure is implemented based on equation (14) and the final value of pheromone $\tau_{r \gamma j F}(N)$ is determined.

Stage 7. Moving to the next iteration. Return of all $z_{\max }$ ants of the colony to the graph's zero node and pheromone updating on every $r \gamma j$-th arc is carried out on the basis of equation (15). Then, transition to Stage 2 is performed.

Stage 8. Completion of the process of designing RB. After completing the design of RB, the fuzzy system parametric optimization as well as development of necessary software and hardware for its further implementation and application may be conducted.

The Fig. 4 shows a block diagram of the proposed ACO based information technology for the designing RBs with finding the optimal consequents for different types fuzzy systems.

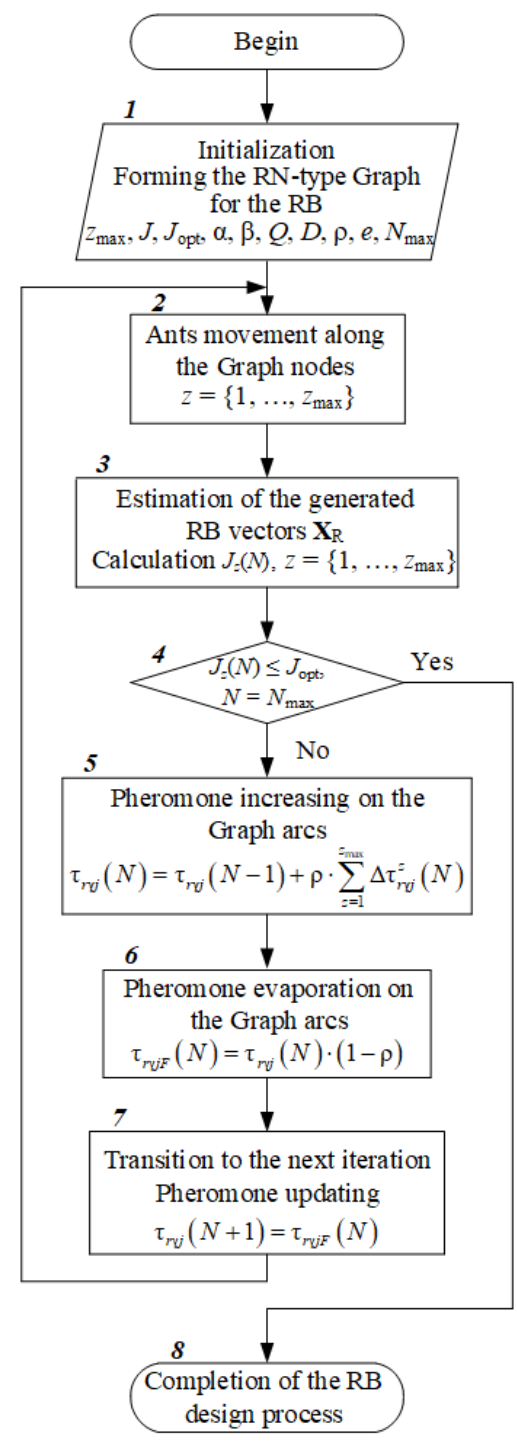

Figure 4. Block diagram of the ACO based information technology for RB design. 
The effective study of the developed information technology is performed in this work on a specific example, in particular, when developing the adaptive fuzzy control system for ship steering device.

\section{DESIGN OF THE RULE BASE FOR ADAPTIVE FUZZY CONTROL SYSTEM OF SHIP STEERING DEVICE}

Ship steering device is a complicated, nonlinear, timevarying, multi-variable hydro-mechatronics system, which performance has a significant influence on the ship normal navigation in various conditions and vitality in whole [66, 67]. One of the most important tasks of automatic control of the steering machine is the tracking control of the ship's rudder angle $\varphi$ by a certain degree. According to the requirements of the Maritime Register the rudder shift time from $0^{\circ}$ to $35^{\circ}$ should be not more than 14 seconds. Thus, the automatic control system of the steering device must provide high quality indicators of the rudder shift angle $\varphi$ control, in particular, high speed with minimal overshoot [66].

The mathematical model of the steering device consists of the following main equations [66]:

$$
\begin{gathered}
m_{\Sigma} \frac{d^{2} Y_{\mathrm{MT}}}{d t^{2}}+k_{\mathrm{FR}} \frac{d Y_{\mathrm{MT}}}{d t}+c_{\mathrm{S}} Y_{\mathrm{MT}}+P_{\mathrm{L}} S_{\mathrm{HC}}=c_{\mathrm{S}} Y_{\mathrm{P}} \\
Q_{\mathrm{V}}=S_{\mathrm{HC}} \frac{d Y_{\mathrm{P}}}{d t}+\frac{\mathrm{v}_{0}}{2 E_{\mathrm{HC}}^{\prime}} \frac{d P_{\mathrm{L}}}{d t} \\
P_{\mathrm{L}}=P_{1}-P_{2}=F_{\mathrm{L}} / S_{\mathrm{HC}} ; \\
Q_{\mathrm{V}}=k_{\mathrm{V}}^{\prime} x_{\mathrm{V}} \sqrt{\frac{P_{\mathrm{in}}-P_{\mathrm{L}}-P_{\mathrm{out}}}{2}} ; \\
k_{\mathrm{V}}^{\prime}=\mu_{\mathrm{V}} \pi d_{\mathrm{V}} k_{\mathrm{f}} \sqrt{2 / \rho_{1}} ; \\
M_{\mathrm{RS}}=5.3 \sin \varphi S_{\mathrm{R}} V_{S}^{2} \\
F_{\mathrm{L}}=\frac{M_{\mathrm{RS}}}{R_{\mathrm{R}}} \\
\varphi=\frac{Y_{\mathrm{MT}}}{R_{\mathrm{R}}}
\end{gathered}
$$

where $m_{\Sigma}$ is the total reduced mass of all moving parts of the hydraulic drive and mechanical transmission of the rudder; $Y_{\mathrm{MT}}$ and $Y_{\mathrm{P}}$ are the linear displacements of the mechanical transmission of the rudder and hydraulic drive plunger; $k_{\mathrm{FR}}$ is the friction coefficient; $c_{\mathrm{s}}$ is the coefficient of stiffness of the connection of the plunger with the mechanical transmission; $P_{\mathrm{L}}$ is the pressure drop in the cavities of the hydraulic cylinder due to the action of the loading force $F_{\mathrm{L}}$; $S_{\mathrm{HC}}$ is the working area of the hydraulic cylinder plunger; $Q_{\mathrm{V}}$ is the value of the working fluid flow through the hydraulic drive valve; $v_{0}$ is the fluid volume contained in the left or right cavity of the hydraulic cylinder; $E_{\mathrm{HC}}^{\prime}$ is the reduced elasticity modulus of the hydraulic cylinder with an elastic support; $P_{1}$ and $P_{2}$ are the pressure values in the left and right cavities of the hydraulic cylinder; $k_{\mathrm{V}}^{\prime}$ is the specific conductivity of the valve windows; $x_{\mathrm{V}}$ is the valve linear displacement; $P_{\text {in }}$ and $P_{\text {out }}$ are the input and output pressure values of the hydraulic drive; $\mu_{\mathrm{V}}$ is the valve flow coefficient; $d_{\mathrm{V}}$ is the valve diameter; $k_{\mathrm{f}}$ is the coefficient of completeness of the use of the valve perimeter by its windows; $\rho_{1}$ is the density of the working fluid of the hydraulic drive; $M_{\mathrm{RS}}$ is the load moment on the rudder stock; $S_{\mathrm{R}}$ is the rudder area; $V_{\mathrm{S}}$ is the ship speed in knots; $R_{\mathrm{R}}$ is the rudder tiller radius.

The given equations describe hydraulic drive, mechanical transmission and the rudder itself as the main parts of the steering device. In turn, the hydraulic drive valve is controlled by the signal $u_{\mathrm{C}}$ and has the following transfer function $W_{\mathrm{V}}(\mathrm{s})$

$$
W_{\mathrm{v}}(\mathrm{s})=\frac{x_{\mathrm{v}}(\mathrm{s})}{u_{\mathrm{C}}(\mathrm{s})}=\frac{k_{\mathrm{V}}}{T_{\mathrm{V}}^{2} \mathrm{~s}^{2}+2 \zeta T_{\mathrm{V}} \mathrm{s}+1},
$$

where $k_{\mathrm{V}}, T_{\mathrm{V}}$ and $\zeta$ are gain, time constant and damping factor of the electrically operated valve, determined by the parameters of its servo drive.

As can be seen from the above equations, the ship's speed $V_{\mathrm{S}}$, as well as the set value of the rudder angle $\varphi_{\mathrm{S}}$, significantly affect the quality indicators of the steering device control. Thus, the use of conventional control laws in the angle control system will not ensure the achievement of sufficiently high quality indicators, in particular, in terms of its speed and overshoot. In turn, to provide high quality indicators of the rudder angle control, as well as to comply with the requirements for speed and overshoot it is advisable to implement adaptive control laws, that take into account change of the ship's speed and angle set values in rather wide ranges. The functional structure of the adaptive control system for the steering machine with fuzzy adaptive device is presented in Fig. 5, where the following notations are accepted: CCS is the control system of the ship's course; ACB is the rudder angle control block; FAD is the fuzzy adaptive device of the angle control system; MB1 and MB2 are the multiplication blocks; SD is the steering device; HD is the hydraulic drive; RMT is the rudder and mechanical transmission; $\varphi_{S}$ and $\varphi_{R}$ are set and real values of the rudder angle; $\varepsilon_{\varphi}$ is the angle control error; $k_{\mathrm{P}}$ and $k_{\mathrm{D}}$ are proportional and differential coefficients, which are subsequently multiplied by the control error and its derivative; $u_{\mathrm{C}}$ is the control signal. 


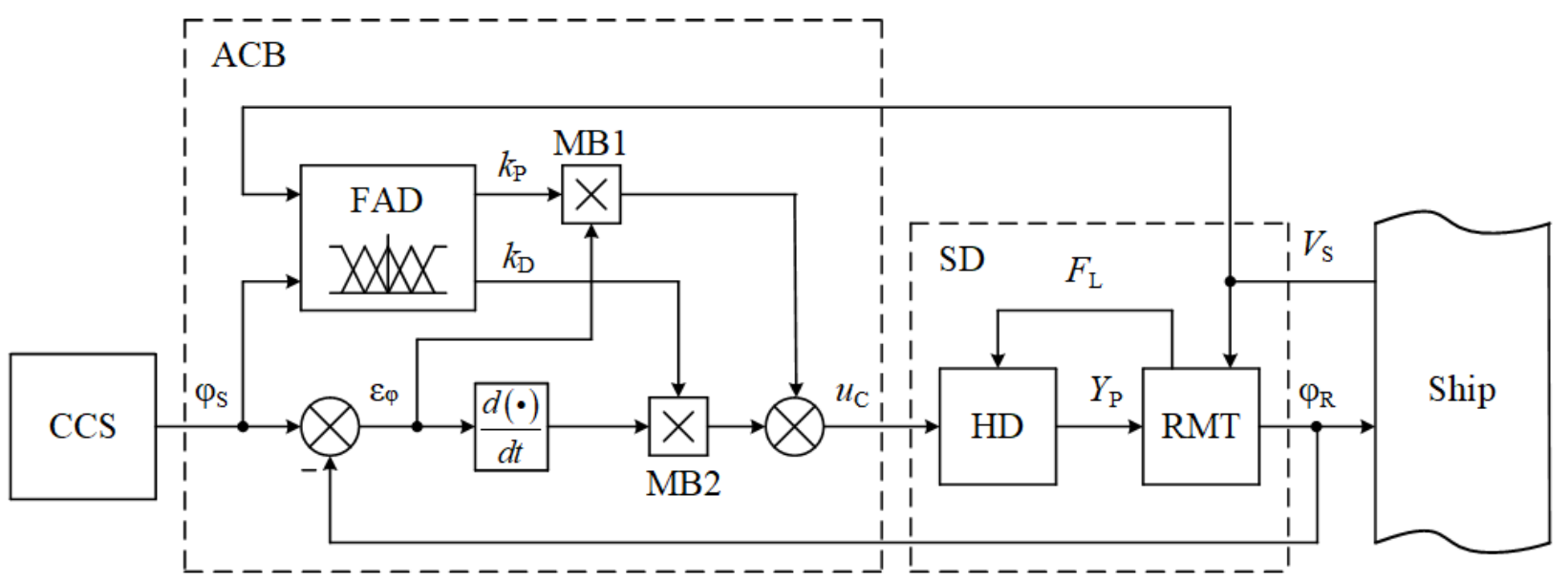

Figure 5. Control system of steering machine with fuzzy adaptive device

The control signal $u_{\mathrm{C}}$ of the given system is calculated by the following equation

$$
u_{\mathrm{C}}=\varepsilon_{\varphi} k_{\mathrm{P}}\left(\varphi_{\mathrm{S}}, V_{\mathrm{S}}\right)+\frac{d \varepsilon_{\varphi}}{d t} k_{\mathrm{D}}\left(\varphi_{\mathrm{S}}, V_{\mathrm{S}}\right)
$$

The fuzzy adaptive device should calculate the optimal values of the proportional and differential coefficients $k_{\mathrm{P}}$ and $k_{\mathrm{D}}$ depending on the taken modulo set angle value $\varphi_{\mathrm{s}}$ of the rudder and the current value of the ship's speed $V_{\mathrm{s}}$. In turn, it is the MIMO fuzzy system that has 2 inputs: $\left|V_{\mathrm{S}}\right|$ and $\left|\varphi_{\mathrm{s}}\right|$, as well as 2 outputs: $k_{\mathrm{P}}$ and $k_{\mathrm{D}}$.

Development of the given FAD for the steering device automatic control system by means of the proposed ACObased information technology is carried out in this paper for the ship with the following parameters: length and water tonnage of the ship are $150 \mathrm{~m}$ and 37000 tons, hydraulic drive power is $160 \mathrm{kWt}$, maximum steering angle of the rudder is $35^{\circ}$, maximum ship's speed is 20 knots.

The designing rule base of the given FAD by means of the presented ACO-based information technology is conducted on the basis of a training sample (Table 1), consisting of sets of values of input variables $\varphi_{\mathrm{S}}$ and $V_{\mathrm{S}}$ and output variables $k_{\mathrm{P}}$ and $k_{\mathrm{D}}$.

Table 1. Training sample for designing RB

\begin{tabular}{|c|c|c|c|c|}
\hline \multirow{2}{*}{$\#$} & \multicolumn{2}{|c|}{ Input variables } & \multicolumn{2}{c|}{ Output variables } \\
\cline { 2 - 5 } & $\boldsymbol{V}_{\mathbf{S}}, \mathbf{k n}$ & $\boldsymbol{\varphi}_{\mathbf{s}}{ }^{\mathbf{}}$ & $\boldsymbol{k}_{\mathbf{P}}$ & $\boldsymbol{k}_{\mathbf{D}}$ \\
\hline 1 & 2 & 7 & 6.32 & 1.93 \\
\hline 2 & 4 & 7 & 6.76 & 2.12 \\
\hline 3 & 6 & 7 & 7.01 & 2.57 \\
\hline 4 & 8 & 7 & 7.62 & 2.68 \\
\hline 5 & 10 & 7 & 7.87 & 3.69 \\
\hline 6 & 12 & 7 & 8.21 & 5.03 \\
\hline 7 & 14 & 7 & 8.65 & 5.67 \\
\hline 8 & 16 & 7 & 9.14 & 8.03 \\
\hline 9 & 18 & 7 & 10.52 & 9.62 \\
\hline 10 & 20 & 7 & 11.69 & 10.74 \\
\hline 11 & 2 & 14 & 6.65 & 2.28 \\
\hline 12 & 4 & 14 & 7.12 & 2.43 \\
\hline 13 & 6 & 14 & 7.44 & 2.97 \\
\hline 14 & 8 & 14 & 8.01 & 3.13 \\
\hline
\end{tabular}

\begin{tabular}{|c|c|c|c|c|}
\hline 15 & 10 & 14 & 8.38 & 4.01 \\
\hline 16 & 12 & 14 & 8.76 & 5.61 \\
\hline 17 & 14 & 14 & 9.13 & 6.12 \\
\hline 18 & 16 & 14 & 9.69 & 8.64 \\
\hline 19 & 18 & 14 & 11.08 & 10.09 \\
\hline 20 & 20 & 14 & 12.21 & 11.23 \\
\hline 21 & 2 & 21 & 7.13 & 3.07 \\
\hline 22 & 4 & 21 & 7.61 & 3.27 \\
\hline 23 & 6 & 21 & 8.02 & 3.59 \\
\hline 24 & 8 & 21 & 8.54 & 3.69 \\
\hline 25 & 10 & 21 & 8.95 & 4.64 \\
\hline 26 & 12 & 21 & 9.32 & 6.13 \\
\hline 27 & 14 & 21 & 9.68 & 6.88 \\
\hline 28 & 16 & 21 & 10.29 & 9.37 \\
\hline 29 & 18 & 21 & 11.63 & 10.74 \\
\hline 30 & 20 & 21 & 12.76 & 11.85 \\
\hline 31 & 2 & 28 & 7.64 & 3.54 \\
\hline 32 & 4 & 28 & 8.12 & 3.87 \\
\hline 33 & 6 & 28 & 8.51 & 4.04 \\
\hline 34 & 8 & 28 & 9.03 & 4.37 \\
\hline 35 & 10 & 28 & 9.47 & 5.12 \\
\hline 36 & 12 & 28 & 9.87 & 6.72 \\
\hline 37 & 14 & 28 & 10.35 & 7.34 \\
\hline 38 & 16 & 28 & 10.87 & 10.02 \\
\hline 39 & 18 & 28 & 12.02 & 11.57 \\
\hline 40 & 20 & 28 & 13.11 & 12.46 \\
\hline 41 & 2 & 35 & 8.08 & 3.96 \\
\hline 42 & 4 & 35 & 8.45 & 4.19 \\
\hline 43 & 6 & 35 & 8.86 & 4.35 \\
\hline 44 & 8 & 35 & 9.32 & 4.95 \\
\hline 45 & 10 & 35 & 9.89 & 5.87 \\
\hline 46 & 12 & 35 & 10.13 & 7.21 \\
\hline 47 & 14 & 35 & 10.93 & 8.92 \\
\hline 48 & 16 & 35 & 11.23 & 10.68 \\
\hline 49 & 18 & 35 & 12.14 & 12.08 \\
\hline 50 & 20 & 35 & 13.54 & 13.22 \\
\hline
\end{tabular}

The given values of the coefficients $k_{\mathrm{P}}$ and $k_{\mathrm{D}}$ (presented in Table 1) are previously obtained using parametric optimization techniques [42] for each separately specified value of set angle $\varphi_{\mathrm{S}}$ of the rudder and ship's speed $V_{\mathrm{S}}$.

When developing the fuzzy adaptive device, five linguistic terms of a trapezoidal type are selected for its first $\left|V_{S}\right|$ and second $\left|\varphi_{S}\right|$ inputs. In turn, for $\left|V_{S}\right|: V L$ - very low; $\mathrm{L}$ - low; M - middle; H - high; VH - very high. For $\left|\varphi_{\mathrm{s}}\right|$ : VS very small; $\mathrm{S}$ - small; $\mathrm{M}$ - middle; $\mathrm{B}$ - big; VB - very big. 
As for FAD outputs, seven terms of a trapezoidal type are selected for $k_{\mathrm{P}}$ and $k_{\mathrm{D}}$ : VS - very small; $\mathrm{S}$ - small; LM - less than middle; $\mathrm{M}$ - middle; $\mathrm{MM}$ - more than middle; $\mathrm{B}$ - big; VB - very big. The appearance of selected LTs with the set vertices is presented in Fig. 6.
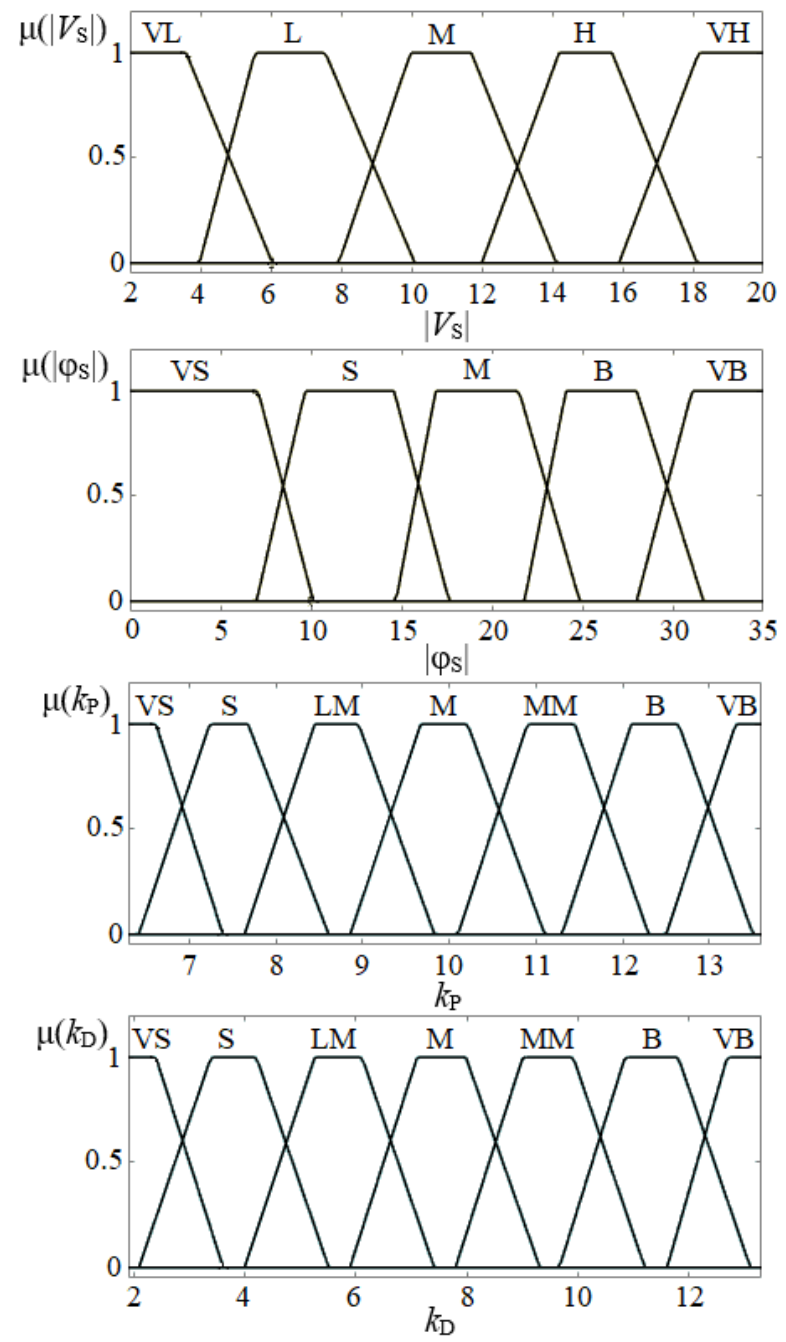

Figure 6. FAD linguistic terms and their parameters.

In this case, the FAD rule base will be full with the number of rules $s=25$ that corresponds to all possible combinations of linguistic terms of the two input variables $\left|V_{\mathrm{S}}\right|$ and $\left|\varphi_{\mathrm{S}}\right|$. The given RB consequents vector $\mathbf{R}_{X}$ for the $x$ th alternative variant is represented as follows

$$
\begin{gathered}
\mathbf{R}_{X}=\left\{L T_{X 11}, L T_{X 12} ; \ldots ; L T_{X r 1}, L T_{X r 2} ; \ldots ;\right. \\
\left.L T_{X 251}, L T_{X 252}\right\}, \\
L T_{X r 1}, L T_{X r 2} \in\{\mathrm{VS}, \mathrm{S}, \mathrm{LM}, \mathrm{M}, \mathrm{MM}, \mathrm{B}, \mathrm{VB}\}, \\
r=\{1,2, \ldots, 25\} .
\end{gathered}
$$

One of the rules of the FAD rule base is represented by the following expression

$$
\begin{gathered}
\text { IF “ }\left|V_{\mathrm{S}}\right|=\mathrm{VL} " \mathrm{AND} "\left|\varphi_{\mathrm{S}}\right|=\mathrm{VS} " \\
\text { THEN “ } k_{\mathrm{P}}=L T_{X 11} " \text { AND “ } k_{\mathrm{D}}=L T_{X 12} ”, \\
L T_{X 11}, L T_{X 12} \in\{\mathrm{VS}, \mathrm{S}, \mathrm{LM}, \mathrm{M}, \mathrm{MM}, \mathrm{B}, \mathrm{VB}\} .
\end{gathered}
$$

At stage 1 of the proposed information technology the $\mathrm{RN}$-type graph is formed, which structure corresponds to the designed rule base of the FAD. The graph consists of one zero node and 50 main nodes $(s m=50)$, sequentially connected by arcs, the total number of which is $350(q=$ $350)$. In turn, each node is connected to the next one on the right by 7 arcs. As the FAD objective function $J$ the MSE is selected, that is calculated as follows

$$
J=\frac{1}{50} \sum_{g=1}^{50}\left(k_{\mathrm{P} g}^{*}-k_{\mathrm{P} g}^{\mathrm{F}}\right)^{2}+\frac{1}{50} \sum_{g=1}^{50}\left(k_{\mathrm{D} g}^{*}-k_{\mathrm{D} g}^{\mathrm{F}}\right)^{2},
$$

where $k_{\mathrm{P} g}^{*}$ and $k_{\mathrm{D} g}^{*}$ are the coefficients actual values from the Table $1 ; k_{\mathrm{P} g}^{\mathrm{F}}$ and $k_{\mathrm{D} g}^{\mathrm{F}}$ are the coefficients values obtained from the FAD outputs at the given values of its inputs; $g$ is the line number of the training sample (Table 1), $g=1,2, \ldots, 50$.

The objective function optimal value is also selected: $J_{\text {opt }}$ $=0.9$.

To study the effectiveness of the proposed ACO-based information technology at its initialization stage, 4 separate colonies with the same number of main ants $z_{\max }=25$ and a different number of elite ants $e$ are created: 1) $e=2$;2) $e=$ 8 ;3) $e=12$; 4) $e=15$. In turn, the designing RB with optimal consequents formation is carried out alternately with the help of each separate colony 5 times (totally 20 experiments) with subsequent selecting the best obtained results. Moreover, for all generated colonies, the same coefficients $\alpha, \beta, Q$ and $\rho$ are set, that have the following values: $\alpha=3, \beta=1, Q=0.8, \rho=$ 0.5 . The values of the relative lengths $D$ for all arcs are set randomly.

All sequential procedures of the design process of the rule base (ant movement, estimation of generated RB vectors, checking for design completion, pheromone increasing, evaporation and updating) are performed in accordance with the main stages of the proposed information technology. In particular, the objective function (28) calculation is performed using all training data from Table 1 at each iteration. The design process completion is determined by the achievement of the maximum number of iterations $N_{\max }=100$.

The results of all 20 experiments obtained for all ants colonies during designing RB for the fuzzy adaptive device are given in Table 2, where the following notations are accepted: $N_{\text {Jopt }}$ is the total number of iterations required to achieve the optimal value of the objective function $J_{\text {opt }} ; J_{\min }$ is the minimal value of the objective function achieved by a colony in 100 iterations when implementing the information technology; $N_{J \min }$ is the total number of iterations required to attain the minimal objective function value $J_{\min }$. In turn, in 
Table 2 best cases for each colony are highlighted in bold.

Table 2. Obtained experimental results.

\begin{tabular}{|c|c|c|c|c|c|}
\hline \multirow{2}{*}{$\#$} & \multirow{2}{*}{$\begin{array}{c}\text { Colony } \\
\text { number }\end{array}$} & \multicolumn{4}{|c|}{ Parameters of Experiments } \\
\cline { 3 - 6 } & 1 & 2 & $\boldsymbol{N}_{\boldsymbol{J} \text { opt }}$ & $\boldsymbol{J}_{\text {min }}$ & $\boldsymbol{N}_{\boldsymbol{J} \text { min }}$ \\
\hline 1 & 1 & 2 & - & 1.64 & 55 \\
\hline 2 & 1 & 2 & - & 1.64 & 78 \\
\hline 3 & 1 & 2 & - & 1.55 & 79 \\
\hline 4 & $\mathbf{1}$ & $\mathbf{2}$ & - & $\mathbf{1 . 4 1}$ & $\mathbf{6 4}$ \\
\hline $\mathbf{5}$ & 2 & 8 & 81 & 0.88 & 81 \\
\hline 6 & 2 & 8 & 92 & 0.86 & 92 \\
\hline 7 & 2 & $\mathbf{8}$ & $\mathbf{7 4}$ & $\mathbf{0 . 8 4}$ & $\mathbf{7 4}$ \\
\hline $\mathbf{8}$ & $\mathbf{2}$ & 8 & - & 1.12 & 76 \\
\hline 9 & 2 & 8 & 77 & 0.85 & 93 \\
\hline 10 & 2 & 12 & 82 & 0.66 & 93 \\
\hline 11 & 3 & $\mathbf{1 2}$ & $\mathbf{5 8}$ & $\mathbf{0 . 5 4}$ & $\mathbf{7 1}$ \\
\hline $\mathbf{1 2}$ & $\mathbf{3}$ & 12 & 73 & 0.59 & 73 \\
\hline 13 & 3 & 12 & 64 & 0.62 & 97 \\
\hline 14 & 3 & 12 & 72 & 0.81 & 72 \\
\hline 15 & 3 & 15 & 94 & 0.82 & 94 \\
\hline 16 & 4 & 15 & 97 & 0.79 & 97 \\
\hline 17 & 4 & 15 & - & 1.04 & 54 \\
\hline 18 & 4 & $\mathbf{1 5}$ & $\mathbf{9 4}$ & $\mathbf{0 . 7 6}$ & $\mathbf{9 4}$ \\
\hline $\mathbf{1 9}$ & $\mathbf{4}$ & 15 & 98 & 0.87 & 98 \\
\hline 20 & 4 & & & & \\
\hline
\end{tabular}

Fig. 7 shows the changing curves of the objective function (28) for best cases for each colony (convergence curves) during the process of designing RB with optimal consequents formation for the FAD based on the proposed information technology.

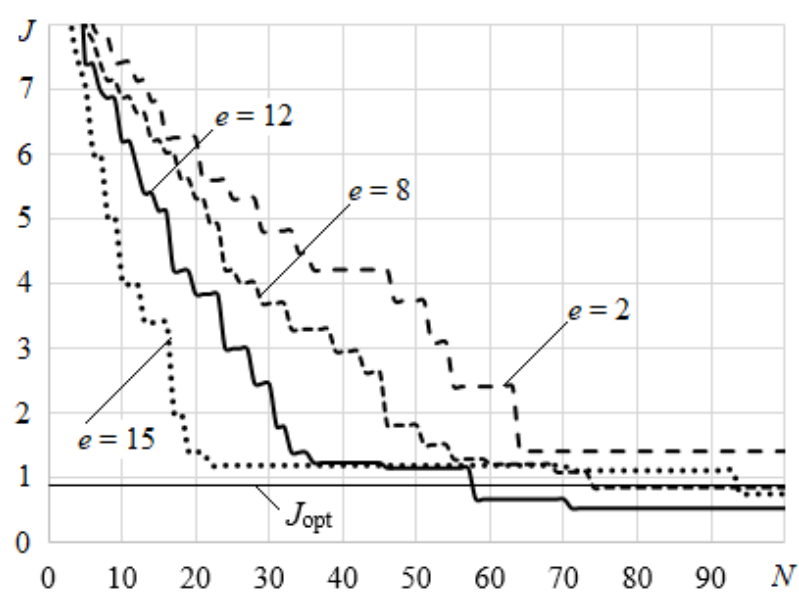

Figure 7. Changing curves of the objective function (28) best values during the design process of RB.

As Table 2 and Fig. 7 show, at small value of the elite agents $(e=2)$ the optimal objective function value has not been reached even once in 100 iterations. At the same time, for large enough values of elite ants $(e=15)$, the optimal value of the objective function $J_{\text {opt }}$ has been reached 4 times out of 5 , but the search process looped in suboptimal solutions for large enough number of iterations and, therefore, more computational costs have been required, than with smaller numbers of elite agents $(e=12)$. Thus, the larger the number of elite ants $e$ in the colony, the faster the FS objective function $J$ decreases at the beginning of the optimization process. So, in general, at increasing the number of elite ants $e$, the convergence rate of the search process also significantly increases, but this number should not be too large to avoid frequent hits in local minima. Thus, analyzing the above results, it may be concluded that $e=12$ is the most suitable value of the elite ants for the colony in this specific case, as it allowed attaining the smallest objective function value $\left(J_{\min }=0.54\right)$ in the smallest value of iterations $\left(N_{J_{\min }}=71\right)$. Also, for this number of elite ants, the optimal value of the objective function $J_{\text {opt }}$ is achieved in the smallest value of iterations $\left(N_{J \text { opt }}=58\right)$.

In turn, the optimal vector of consequents $\mathbf{R}_{\text {opt }}$ synthesized using the third colony with 12 elite ants (the best variant) has the form:

$\mathbf{R}_{\mathrm{opt}}=(\mathrm{VS}, \mathrm{VS} ; \mathrm{VS}, \mathrm{VS} ; \mathrm{S}, \mathrm{S} ; \mathrm{S}, \mathrm{S} ; \mathrm{LM}, \mathrm{S} ; \mathrm{S}, \mathrm{VS} ; \mathrm{S}, \mathrm{S} ;$ LM, S; LM, S; LM, LM; S, S; LM, LM; LM, LM; M, M; M, M; LM, M; M, M; M, M; M, MM; MM, MM; MM, MM; B, $\mathrm{B} ; \mathrm{B}, \mathrm{B} ; \mathrm{B}, \mathrm{VB}$; VB, VB).

Moreover the obtained rule base of FAD with the given consequents vector $\mathbf{R}_{\text {opt }}$ is presented in Table 3 .

Table 3. RB synthesized by means of the $3^{\text {rd }}$ colony

\begin{tabular}{|c|c|c|c|c|}
\hline \multirow{2}{*}{$\begin{array}{c}\text { Rule } \\
\text { number }\end{array}$} & \multicolumn{4}{|c|}{ Input and output variables } \\
\hline & $V_{\mathrm{S}}, \mathbf{k n}$ & $\varphi_{\mathrm{s}},{ }^{0}$ & $k_{\mathrm{P}}$ & $k_{\mathrm{D}}$ \\
\hline 1 & VL & VS & VS & VS \\
\hline 2 & VL & $\mathrm{S}$ & VS & VS \\
\hline 3 & VL & $\mathrm{M}$ & $\mathrm{S}$ & $\mathrm{S}$ \\
\hline 4 & VL & B & $\mathrm{S}$ & $S$ \\
\hline 5 & VL & VB & LM & $\mathrm{S}$ \\
\hline 6 & $\mathrm{~L}$ & VS & $\mathrm{S}$ & VS \\
\hline 7 & $\mathrm{~L}$ & $\mathrm{~S}$ & $\mathrm{~S}$ & $\mathrm{~S}$ \\
\hline 8 & $\mathrm{~L}$ & $\mathrm{M}$ & LM & $\mathrm{S}$ \\
\hline 9 & $\mathrm{~L}$ & $\mathrm{~B}$ & LM & $\mathrm{S}$ \\
\hline 10 & $\mathrm{~L}$ & VB & LM & LM \\
\hline 11 & M & VS & $\mathrm{S}$ & $\mathrm{S}$ \\
\hline 12 & $\mathrm{M}$ & $\mathrm{S}$ & LM & LM \\
\hline 13 & M & $\mathrm{M}$ & LM & LM \\
\hline 14 & $\mathrm{M}$ & B & $\mathrm{M}$ & $\mathrm{M}$ \\
\hline 15 & $\mathrm{M}$ & VB & $\mathrm{M}$ & $\mathrm{M}$ \\
\hline 16 & $\mathrm{H}$ & VS & LM & $\mathrm{M}$ \\
\hline 17 & $\mathrm{H}$ & $\mathrm{S}$ & $\mathrm{M}$ & M \\
\hline 18 & $\mathrm{H}$ & M & $\mathrm{M}$ & $\mathrm{M}$ \\
\hline 19 & $\mathrm{H}$ & $\mathrm{B}$ & $\mathrm{M}$ & MM \\
\hline 20 & $\mathrm{H}$ & VB & MM & $\mathrm{MM}$ \\
\hline 21 & $\mathrm{VH}$ & VS & MM & MM \\
\hline 22 & VH & $\mathrm{S}$ & B & B \\
\hline 23 & $\mathrm{VH}$ & $\mathrm{M}$ & B & $\mathrm{B}$ \\
\hline 24 & $\mathrm{VH}$ & $\mathrm{B}$ & $\mathrm{B}$ & VB \\
\hline 25 & VH & VB & VB & VB \\
\hline
\end{tabular}

To evaluate the efficiency of the rudder angle adaptive control system, as well as the proposed ACO-based information technology for synthesizing the rule base of its FAD, the rudder shift transients have been simulated at different modes. In particular, Fig. 8 and 9, respectively, show the rudder shift transients from $0^{\circ}$ to $35^{\circ}$ for two different cases. 


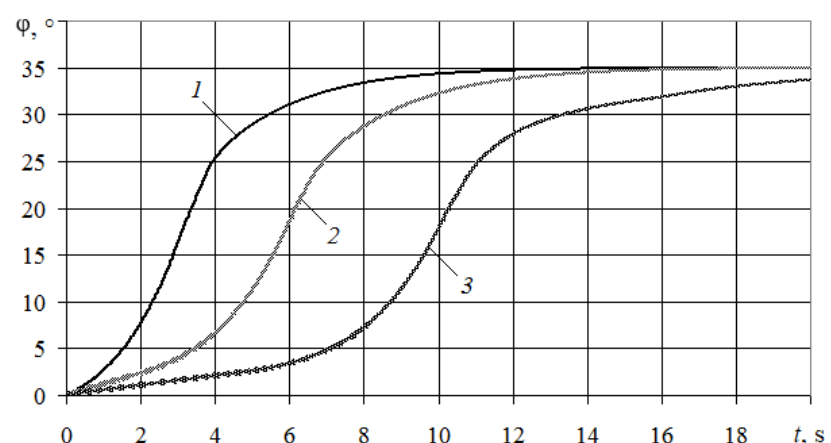

Figure 8. Rudder shift transients for conventional control system.

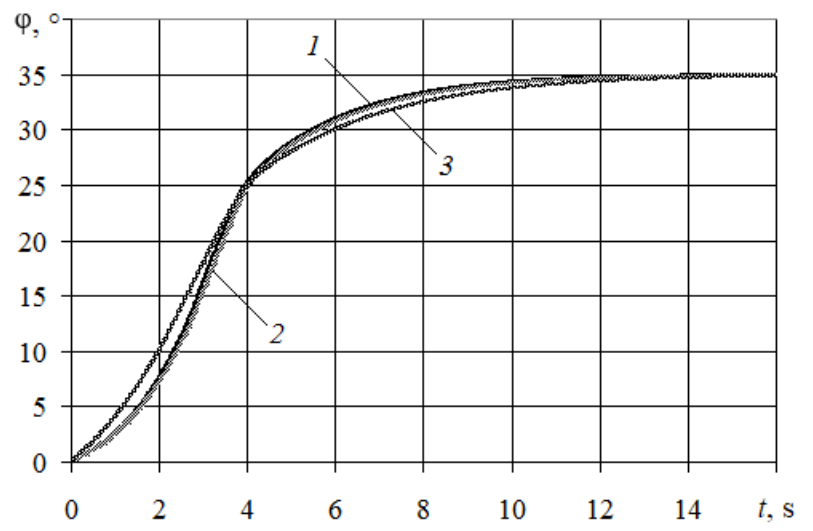

Figure 9. Rudder shift transients for adaptive control system with FAD.

In turn, in the first case (Fig. 8) the control system with conventional proportional-differential control law is applied (the coefficients $k_{\mathrm{P}}$ and $k_{\mathrm{D}}$ are optimally tuned for the rudder angle set value $\varphi_{S}=35^{\circ}$ and the ship's speed $V_{S}=2 \mathrm{kn}$.).

In the second case (Fig. 9) the developed adaptive control system with FAD (Fig. 5) is used, whose rule base is designed by means of the proposed ACO-based information technology. For both cases (Fig. 8 and 9), the simulation results are obtained for three different values of the ship's speed: 1) $V_{\mathrm{S}}=2 \mathrm{kn}$; 2) $V_{\mathrm{S}}=10 \mathrm{kn}$; 3) $V_{\mathrm{S}}=20 \mathrm{kn}$.

Fig. 8 shows that the conventional control system cannot provide high enough quality indicators of control at the values of the ship's speed $V_{\mathrm{S}}=10 \mathrm{kn}$ and $V_{\mathrm{S}}=20 \mathrm{kn}$. Namely, the rudder shift time from $0^{\circ}$ to $35^{\circ}$ is 14.9 seconds at $V_{\mathrm{S}}=10$ $\mathrm{kn}$ and more than 20 seconds at $V_{\mathrm{S}}=20 \mathrm{kn}$ that does not meet the requirements of the Maritime Register. In turn, the results presented in Fig. 9 show that developed adaptive control system with FAD allows achieving high enough quality indicators of rudder angle control. In particular, the rudder shift time is less than 14 seconds for all values of the ship's speed $V_{\mathrm{S}}$ at zero overshoot. Moreover, the transients graphs change insignificantly with a significant change in the ship's speed (from $2 \mathrm{kn}$ to $20 \mathrm{kn}$ ), which indicates the high robust properties of the developed adaptive system with FAD. Also, to find the optimal consequents vector $\mathbf{R}_{\text {opt }}$ of the rule base for the given fuzzy adaptive device using the proposed ACO- based information technology, minor computational costs were required that confirms its high efficiency.

In turn, if there is a need for additional improvement of the control quality indicators of the given rudder adaptive control system, after designing of the FAD rule base with the optimal consequents vector Ropt using the proposed information technology, it is possible to perform further parametric optimization of its linguistic terms by means of specific optimization techniques [36, 42].

\section{CONCLUSIONS}

The development and research of the universal information technology for designing RBs with the formation of optimal consequents for different types of FSs based on ant colony optimization techniques is presented in this work. The proposed ACO-based information technology allows effectively synthesizing rule bases of various dimensions both for the MISO and MIMO fuzzy systems taking into account the particular features of the RB consequents formation in the conditions of insufficient initial information. It uses the "Rule-Node" concept for constructing the rule base graph in the optimization propocess, that gives the opportunity to spend insignificant computational and time costs when developing fairly complex RBs.

To evaluate the effectiveness of the presented ACObased information technology the design of the rule base for the fuzzy adaptive device of the control system for the ship steering machine is carried out in this work. Herewith, to study the peculiarities of this information technology the design of the FAD rule base with the optimal consequents vector $\mathbf{R}_{\mathrm{opt}}$ is conducted at different number of elite ants $e$ in the colony. The analysis of the experiment results showes that $e=12$ is the most suitable value of the elite ants in the colony for this specific case, as it allows finding the best solution $\left(J_{\min }=0.54\right)$ at the lowest computational costs $\left(N_{J \min }\right.$ =71). In turn, the computer simulations results of the rudder shift transients show that adaptive control system with developed FAD provides achievement of enough high quality indicators of rudder angle control (rudder shift time is less than 14 seconds at zero overshoot) at significant change in the ship's speed (from $2 \mathrm{kn}$ to $20 \mathrm{kn}$ ). Thus, the application of the proposed ACO-based information technology, in this case, allows designing effective RB with optimal consequents vector $\mathbf{R}_{\mathrm{opt}}$ by means of minor computational and time costs that, in turn, confirms its high efficiency.

Further research should be related towards the application of the proposed ACO-based information technology for designing hierarchical fuzzy systems of various configurations.

\section{References}

[1] L. A. Zadeh, A. M. Abbasov, R. R. Yager, S. N. Shahbazova, M. Z. Reformat, Eds., "Recent developments and new directions in soft 
computing," STUDFUZ 317, Cham: Springer, 2014, 466 p. https://doi.org/10.1007/978-3-319-06323-2.

[2] Y. P. Kondratenko, O. V. Korobko, O. V. Kozlov, "Synthesis and optimization of fuzzy controller for thermoacoustic plant," Lotfi A. Zadeh et al. (Eds.), Recent Developments and New Direction in SoftComputing Foundations and Applications, Studies in Fuzziness and Soft Computing, vol. 342, Berlin, Heidelberg: Springer-Verlag, 2016, pp. 453-467. https://doi.org/10.1007/978-3-319-32229-2_31.

[3] M. Jamshidi, V. Kreinovich, J. Kacprzyk, Eds., "Advance Trends in Soft Computing," STUDFUZ 312, Cham: Springer-Verlag, 2013, 468 p. https://doi.org/10.1007/978-3-319-03674-8.

[4] L. A. Zadeh, "The role of fuzzy logic in modeling, identification and control," Modeling Identification and Control, vol. 15, issue 3, pp. 191-203, 1994. https://doi.org/10.4173/mic.1994.3.9.

[5] E. H. Mamdani, "Application of fuzzy algorithms for control of simple dynamic plant," Proceedings of IEEE, vol. 121, pp. 1585-1588, 1974. https://doi.org/10.1049/piee.1974.0328.

[6] B. Kosko, "Fuzzy systems as universal approximators," IEEE Transactions on Computers, vol. 43, no. 11, pp. 1329-1333, 1994. https://doi.org/10.1109/12.324566.

[7] Y. P. Kondratenko, O. V. Korobko, O. V. Kozlov, "Frequency tuning algorithm for loudspeaker driven thermoacoustic refrigerator optimization," Lecture Notes in Business Information Processing: Modeling and Simulation in Engineering, Economics and Management. - K. J. Engemann, A. M. Gil-Lafuente, J. M. Merigo (Eds.), vol. 115, Berlin, Heidelberg: Springer-Verlag, 2012, pp. 270279. https://doi.org/10.1007/978-3-642-30433-0_27.

[8] V. M. Kuntsevich, et al. (Eds)., Control Systems: Theory and Applications, Series in Automation, Control and Robotics, River Publishers, 2018.

[9] Y. P. Kondratenko, O. V. Kozlov, "Mathematic modeling of reactor's temperature mode of multiloop pyrolysis plant," Modeling and Simulation in Engineering, Economics and Management, Lecture Notes in Business Information Processing, vol. 115, 2012, pp. 178187. https://doi.org/10.1007/978-3-642-30433-0_18.

[10] T. Bora, P. Chatterjee, S. Ghosh, "Fuzzy logic based control of variable wind energy system," Proceedings of the 2020 5th IEEE International Conference on Recent Advances and Innovations in Engineering (ICRAIE), Jaipur, India, pp. 1-5, 2020. https://doi.org/10.1109/ICRAIE51050.2020.9358376.

[11] A. Piegat, Fuzzy Modeling and Control, Springer, Heidelberg, 2001, 728 p. https://doi.org/10.1007/978-3-7908-1824-6.

[12] V. Uslan, H. Seker, R. John, "Overlapping clusters and support vector machines based interval type-2 fuzzy system for the prediction of peptide binding affinity," IEEE Access, vol.7, pp. 49756-49764, 2019. https://doi.org/10.1109/ACCESS.2019.2910078.

[13] A. Jain, M. B. Jain, "Fuzzy modeling and similarity based short term load forecasting using evolutionary particle swarm optimization," Proceedings of the 2012 IEEE Power and Energy Society General Meeting, 2012, pp. 1-1. https://doi.org/10.1109/PESGM.2012.6345769.

[14] J. M. Mendel, Uncertain Rule-based Fuzzy Systems, Introduction and New Directions, Second Edition, Springer International Publishing, 2017, 684 p. https://doi.org/10.1007/978-3-319-51370-6.

[15] W. A. Lodwick, J. Kacprzhyk, Eds., "Fuzzy optimization," STUDFUZ, vol. 254, Berlin, Heidelberg: Springer-Verlag, 2010.

[16] O. Kosheleva, V. Kreinovich, "Why Bellman-Zadeh approach to fuzzy optimization," Appl. Math. Sci., vol. 12, pp. 517-522, 2018. https://doi.org/10.12988/ams.2018.8456.

[17] G. M. Méndez, P. N. M. Dorantes, A. M. Santoyo, "Interval type-2 fuzzy logic systems optimized by central composite design to create a simplified fuzzy rule base in image processing for quality control application," The International Journal of Advanced Manufacturing Technology, vol. 102, no. 9-12, pp. 3757-3766, 2019. https://doi.org/10.1007/s00170-019-03354-5.

[18] D. Simon, Evolutionary Optimization Algorithms: Biologically Inspired and Population-based Approaches to Computer Intelligence, John Wiley \& Sons, 2013, 772 p.
[19] A. Melendez, O. Castillo, "Evolutionary optimization of the fuzzy integrator in a navigation system for a mobile robot," Recent Advances on Hybrid Intelligent Systems, pp. 21-31, 2013. https://doi.org/10.1007/978-3-642-33021-6_2.

[20] J. Zhu, F. Lauri, A. Koukam, V. Hilaire, "Fuzzy logic control optimized by artificial immune system for building thermal condition," Siarry P., Idoumghar L., Lepagnot J. (eds) Swarm Intelligence Based Optimization, ICSIBO 2014, Lecture Notes in Computer Science, vol. 8472, Springer, Cham, 2014, pp. 42-49. https://doi.org/10.1007/978-3-319-12970-9_5.

[21] I. Boussaï, J. Lepagnot, P. Siarry, "A survey on optimization metaheuristics," Information Science, vol. 237, pp. 82-117, 2013. https://doi.org/10.1016/j.ins.2013.02.041.

[22] A. Gogna, A. Tayal. "Metaheuristics: review and application," Journal of Experimental and Theoretical Artificial Intelligence, vol. 25, pp. 503-526, 2013. https://doi.org/10.1080/0952813X.2013.782347.

[23] C. Blum, J. Puchinger, G. R. Raidl, A. Roli, "Hybrid metaheuristics in combinatorial optimization: a survey," Applied Soft Computing, vol. 11, pp. 4135-4151, 2011. https://doi.org/10.1016/j.asoc.2011.02.032.

[24] T. Takagi, M. Sugeno, "Fuzzy identification of systems and its applications to modeling and control," IEEE Transactions on Systems, Man, and Cybernetics, SMC-15, no. 1, pp. 116-132, 1985. https://doi.org/10.1109/TSMC.1985.6313399.

[25] R.-C. Roman, R.-E. Precup, C.-A. Bojan-Dragos, A.-I. SzedlakStinean, "Combined model-free adaptive control with fuzzy component by virtual reference feedback tuning for tower crane systems," Procedia Computer Science, vol. 162, pp. 267-274, 2019. https://doi.org/10.1016/j.procs.2019.11.284.

[26] Z. Xiao, J. Guo, H. Zeng, P. Zhou, S. Wang, "Application of fuzzy neural network controller in hydropower generator unit," $J$. Kybernetes, vol. 38 , no. 10, pp. 1709-1717, 2009. https://doi.org/10.1108/03684920910994079.

[27] M. I. Fadholi, Suhartono, P. S. Sasongko, Sutikno, “Autonomous pole balancing design in quadcopter using behaviour-based intelligent fuzzy control," Proceedings of the 2018 2nd International Conference on Informatics and Computational Sciences (ICICoS), Semarang, Indonesia, $\quad$ pp. $1-6, \quad 2018$. https://doi.org/10.1109/ICICOS.2018.8621736.

[28] Y. P. Kondratenko, O. V. Kozlov, "Mathematical model of ecopyrogenesis reactor with fuzzy parametrical identification," Recent Developments and New Direction in Soft-Computing Foundations and Applications, Studies in Fuzziness and Soft Computing, Vol. 342, Lotfi A. Zadeh et al. (Eds.). Berlin, Heidelberg: Springer-Verlag, 2016, pp. 439-451. https://doi.org/10.1007/978-3-319-32229-2 30.

[29] Y. P. Kondratenko, A. V. Kozlov, "Generation of rule bases of fuzzy systems based on modified ant colony algorithms," Journal of Automation and Information Sciences, vol. 51, issue 3, New York: Begel House Inc., pp. 4-25, 2019. https://doi.org/10.1615/JAutomatInfScien.v51.i3.20.

[30] G. Ruiz-García, H. Hagras, H. Pomares, I. R. Ruiz, "Toward a fuzzy logic system based on general forms of interval type-2 fuzzy sets," IEEE Transactions on Fuzzy Systems, vol. 27, no. 12, pp. 2381-2395, 2019. https://doi.org/10.1109/TFUZZ.2019.2898582.

[31] P. Hajek, V. Olej, "Interval-valued intuitionistic fuzzy inference system for supporting corporate financial decisions," Proceedings of the 2018 IEEE International Conference on Fuzzy Systems (FUZZIEEE), 2018, pp. 1-7. https://doi.org/10.1109/FUZZIEEE.2018.8491620.

[32] D. Driankov, H. Hellendoorn, M. Reinfrank, An Introduction to fuzzy Control, Springer Science \& Business Media, 2013.

[33] Y. P. Kondratenko, A. V. Kozlov, "Parametric optimization of fuzzy control systems based on hybrid particle swarm algorithms with elite strategy," Journal of Automation and Information Sciences, vol. 51, $\begin{array}{lllll}\text { issue } 12, \quad \text { New } & \text { pp. 25-45, }\end{array}$ https://doi.org/10.1615/JAutomatInfScien.v51.i12.40.

[34] S. Khan, et al., "Design and implementation of an optimal fuzzy logic controller using genetic algorithm," Journal of Computer Science, vol. 
$\begin{array}{rrrrrr}4, & \text { no } & 10, & \text { pp } & 799-806, & 2008 .\end{array}$ https://doi.org/10.3844/jessp.2008.799.806.

[35] D. Simon, "Hळ estimation for fuzzy membership function optimization," International Journal of Approximate Reasoning, 40, pp. 224-242, 2005. https://doi.org/10.1016/j.ijar.2005.04.002.

[36] F. Valdez, P. Melin and O. Castillo, "Evolutionary method combining particle swarm optimization and genetic algorithms using fuzzy logic for decision making," Proceedings of the 2009 IEEE International Conference on Fuzzy Systems, 2009, pp. 2114-2119. https://doi.org/10.1109/FUZZY.2009.5277165.

[37] O. Kozlov, G. Kondratenko, Z. Gomolka, Y. Kondratenko, "Synthesis and optimization of green fuzzy controllers for the reactors of the specialized pyrolysis plants," Kharchenko V., Kondratenko Y., Kacprzyk J. (eds), Green IT Engineering: Social, Business and Industrial Applications, Studies in Systems, Decision and Control, vol 171, Springer, Cham, 2019, pp. 373-396. https://doi.org/10.1007/9783-030-00253-4 16.

[38] H. Ishibuchi, T. Yamamoto, "Fuzzy rule selection by multi-objective genetic local search algorithms and rule evaluation measures in data mining," Fuzzy Sets and Systems, vol. 141, no. 1, pp. 59-88, 2004. https://doi.org/10.1016/S0165-0114(03)00114-3.

[39] B. Jayaram, "Rule reduction for efficient inferencing in similarity based reasoning," International Journal of Approximate Reasoning, vol. 48, no. 1, pp. 156-173, 2008. https://doi.org/10.1016/j.ijar.2007.07.009.

[40] P. C. Shill, Y. Maeda, K. Murase, "Optimization of fuzzy logic controllers with rule base size reduction using genetic algorithms," Proceedings of the 2013 IEEE Symposium on Computational Intelligence in Control and Automation (CICA), Singapore, 2013, pp. 57-64. https://doi.org/10.1109/CICA.2013.6611664.

[41] C. Li, H. Zhao, S. Zhen, Y. -H. Chen, "Control design with optimization for fuzzy steering-by-wire system based on nash game theory," IEEE Transactions on Cybernetics, pp. 1-10, 2021. https://doi.org/10.1109/TCYB.2021.3050509.

[42] L. D. Seixas, H. G. Tosso, F. C. Corrêa, J. J. Eckert, "Particle swarm optimization of a fuzzy controlled hybrid energy storage system HESS," Proceedings of the 2020 IEEE Vehicle Power and Propulsion Conference (VPPC), Gijon, Spain, pp. 1-6. 2020. https://doi.org/10.1109/VPPC49601.2020.9330939.

[43] C. Juang, C. Hsiao and C. Hsu, "Hierarchical cluster-based multispecies particle-swarm optimization for fuzzy-system optimization," IEEE Transactions on Fuzzy Systems, vol. 18, no. 1, pp. 14-26, 2010. https://doi.org/10.1109/TFUZZ.2009.2034529

[44] W. Pedrycz, K. Li, M. Reformat, "Evolutionary reduction of fuzzy rule-based models," Fifty Years of Fuzzy Logic and its Applications, STUDFUZ, vol. 326, 2015, Cham: Springer, pp. 459-481. https://doi.org/10.1007/978-3-319-19683-1_23.

[45] R. Alcalá, J. Alcalá-Fdez, M. J. Gacto, F. Herrera, "Rule base reduction and genetic tuning of fuzzy systems based on the linguistic 3-tuples representation," Soft Computing, vol. 11, no. 5, pp. 401-419, 2007. https://doi.org/10.1007/s00500-006-0106-2.

[46] A. Nabi, N.A. Singh, "Particle swarm optimization of fuzzy logic controller for voltage sag improvement," Proceedings of 2016 3rd International Conference on Advanced Computing and Communication Systems (ICACCS), vol. 01, 2016, pp. 1-5. https://doi.org/10.1109/ICACCS.2016.7586345.

[47] M. Dorigo, M. Birattari, Ant Colony Optimization, Encyclopedia of Machine Learning, Sammut C., Webb G.I. (eds.), Springer, Boston, MA, 2011. https://doi.org/10.1007/978-0-387-30164-8_22.

[48] N. Quijano, K. M. Passino, Honey Bee Social Foraging Algorithms for Resource Allocation: Theory and Application, Columbus: Publishing house of the Ohio State University, 39 p., 2007. https://doi.org/10.1109/ACC.2007.4282168.

[49] S. Vaneshani, H. Jazayeri-Rad, "Optimized fuzzy control by particle swarm optimization technique for control of CSTR," International Journal of Electrical and Computer Engineering, vol. 5, no. 11, pp. 1243-1248, 2011.
[50] D. H. Kim, C. H. Cho, "Bacterial foraging based neural network fuzzy learning," Proceedings of the 2nd Indian International Conference on Artificial Intelligence (IICAI'2005), Pune, pp. 2030-2036, 2005.

[51] L. Fan, E. M. Joo, "Design for auto-tuning PID controller based on genetic algorithms," Proceedings of the 4th IEEE Conference on Industrial Electronics and Applications ICIEA '2009, 2009, pp. 1924 1928. https://doi.org/10.1109/ICIEA.2009.5138538.

[52] F. L. Minku, T. Ludermir, "Evolutionary strategies and genetic algorithms for dynamic parameter optimization of evolving fuzzy neural networks," Proceedings of the 2005 IEEE Congress on Evolutionary Computation, vol. 3, 2005, pp. 1951-1958.

[53] O. Castillo, P. Ochoa, J. Soria, "Differential evolution with fuzzy logic for dynamic adaptation of parameters in mathematical function optimization," Angelov P., Sotirov S. (eds), Imprecision and Uncertainty in Information Representation and Processing. Studies in Fuzziness and Soft Computing, vol. 332. Springer, Cham, 2016, pp. 361-374. https://doi.org/10.1007/978-3-319-26302-1_21.

[54] D. Simon, "Biogeography-based optimization," IEEE Transactions on Evolutionary Computation, vol. 12, issue 6, pp. 702-713, 2008. https://doi.org/10.1109/TEVC.2008.919004.

[55] R. T. Alves, M. R. Delgado, H. S. Lopes, A. A. Freitas, "An artificial immune system for fuzzy-rule induction in data mining," Yao X. et al. (eds), Parallel Problem Solving from Nature - PPSN VIII. PPSN 2004. Lecture Notes in Computer Science, vol. 3242. Springer, Berlin, Heidelberg, 2004, pp. 1011-1020. https://doi.org/10.1007/978-3-54030217-9_102.

[56] J.-J. Qin, X.-X. Zhang, G.-T. Cao, "Particle swarm optimization based scaling factor tuning for 3-D fuzzy logic controller," Proceedings of the 2010 Seventh International Conference on Fuzzy Systems and Knowledge Discovery, 2010, pp. 711-715. https://doi.org/10.1109/FSKD.2010.5569388.

[57] Y. P. Kondratenko, O. V. Kozlov, O. V. Korobko, "Two modifications of the automatic rule base synthesis for fuzzy control and decision making systems," J. Medina et al. (Eds), Information Processing and Management of Uncertainty in Knowledge-Based Systems: Theory and Foundations, 17th International Conference, IPMU 2018, Cadiz, Spain, Proceedings, Part II, CCIS 854, Springer International Publishing AG, 2018, pp. 570-582. https://doi.org/10.1007/978-3319-91476-3 47.

[58] R. Putha, L. Quadrifoglio, E. Zechman, "Comparing ant colony optimization and genetic algorithm approaches for solving traffic signal coordination under oversaturation conditions," Comput. Aided Civ. Infrastruct. Eng., vol. 27, pp. 14-28, 2012. https://doi.org/10.1111/j.1467-8667.2010.00715.X.

[59] R. Gan, Q. Guo, H. Chang, Y. Yi, "Improved ant colony optimization algorithm for the traveling salesman problems," Journal of Systems Engineering and Electronics, pp. 329-333, 2010. https://doi.org/10.3969/j.issn.1004-4132.2010.02.025.

[60] R.-M. Chen, Y.-M. Shen, C.-T. Wang, "Ant colony optimization inspired swarm optimization for grid task scheduling," Proceedings of the 2016 International Symposium on Computer, Consumer and Control (IS3C), 2016, pp. 461-464. https://doi.org/10.1109/IS3C.2016.122.

[61] Q. Chengming, "Vehicle routing optimization in logistics distribution using hybrid ant colony algorithm," TELKOMNIKA Indonesian Journal of Electrical Engineering, vol. 11, no. 9, pp. 5308-5315, 2013. https://doi.org/10.11591/telkomnika.v11i9.3284

[62] B. Benhala, A. Ahaitouf, M. Fakhfakh, A. Mechaqrane, "New adaptation of the ACO algorithm for the analog circuits design optimization," International Journal of Computer Science (IJCSI), vol. 9, no. 3, pp. 360-367, 2012.

[63] Y. Khaluf, S. Gullipalli, "An efficient ant colony system for edge detection in image processing," Proceedings of the European Conference on Artificial Life, pp. 398-405, 2015.

[64] C.-F. Juang, H.-J. Huang, C.-M. Lu, "Fuzzy controller design by ant colony optimization," Proceedings of the 2007 IEEE International Fuzzy Systems Conference, London, UK, 2007, pp. 1-5. https://doi.org/10.1109/FUZZY.2007.4295335. 
[65] X. Yalang, S. Shiyu and H. Xin, "Optimization design of fuzzy controller based on improved ant colony algorithm," Proceedings of the 2011 First International Conference on Instrumentation, Measurement, Computer, Communication and Control, Beijing, 2011, pp. 875-878. https://doi.org/10.1109/IMCCC.2011.221.

[66] G. Wei, Z. Xian-ku and W. Xin-ping, "Concise nonlinear adaptive robust control of ship steering systems," Proceedings of the 2009 IEEE International Conference on Automation and Logistics, Shenyang, 2009, pp. 343-346. https://doi.org/10.1109/ICAL.2009.5262902.

[67] J. Zhao, Y. Fu, J. Fu and G. Liu, "Fuzzy CMAC compound control of hydraulic servo actuation for ship steering system," Proceedings of the 2017 IEEE International Conference on Mechatronics and Automation (ICMA), Takamatsu, 2017, pp. 1792-1797. https://doi.org/10.1109/ICMA.2017.8016089.

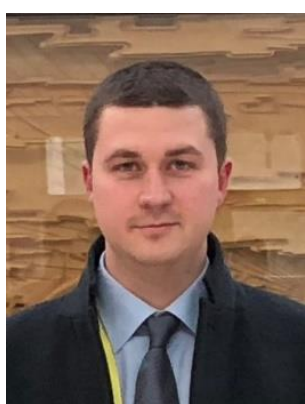

OLEKSIY KOZLOV, Ph.D., Associate Professor, Doctorant and Associate Professor of the Department of Intelligent Information Systems at Petro Mohyla Black Sea National University (PMBSNU), Ukraine. He has received master degree in electromechanics (2011) from Admiral Makarov National University of Shipbuilding and Ph.D. degree in control processes automation (2014) from Odessa National Polytechnic University. Since 2011 took part in the implementation of a number of international and state projects related to automation of complex industrial plants, information technologies, intelligent control systems, robotics and Internet of things. His research interests include automation, intelligent information and control systems, fuzzy logic, bioinspired optimization techniques, robotics. 